\title{
Equilibrium Transitions from Non Renewable Energy to Renewable Energy under Capacity Constraints ${ }^{12}$
}

\author{
Jean-Pierre Amigues ${ }^{3}$, Alain Ayong Le Kama ${ }^{4}$, \\ and Michel Moreaux ${ }^{5}$
}

March 2015

\footnotetext{
${ }^{1}$ The research leading to these results has received funding from European Community's Seventh Framework Programme (FP7/2007-2013) under grant agreement n 266992 - Global-IQ "Impacts Quantification of global changes".

${ }^{2}$ We thank the editor and three referees for their valuable comments. We also thank Steve Salant for his precious advice which allowed us to considerably improve the present paper. Jean-Pierre Amigues also thanks all the participants in the INRA-Paris seminar, the Paris I environmental economics seminar and the EAERE Rome conference for their fruitful comments. The usual disclaimer applies.

${ }^{3}$ Toulouse School of Economics (INRA, IDEI, LERNA), 21 allée de Brienne, 31000 Toulouse, France.

${ }^{4}$ Université de Paris-Ouest Nanterre-La Défense, 200 Avenue de la République, Bât. G - 92001 Nanterre Cedex France

${ }^{5}$ Toulouse School of Economics (IDEI, IUF, LERNA), 21 allée de Brienne, 31000 Toulouse, France.
} 


\begin{abstract}
We study the transition between non renewable and renewable energy sources with adjustment costs over the production capacity of renewable energy. Assuming constant variable marginal costs for both energy sources, convex adjustment costs and a more expensive renewable energy, we show the following. With sufficiently abundant non renewable energy endowments, the dynamic equilibrium path is composed of a first time phase of only non renewable energy use followed by a transition phase substituting progressively renewable energy to non renewable energy before a last time phase of only renewable energy use. The investment into renewable energy may either begin before actual production of renewable energy or be delayed until the energy price achieves a sufficient gap with respect to the renewable energy cost. With an initially abundant non renewable resource, the features of the transition between non renewable and renewable energy do not depend upon the initial resource stock.
\end{abstract}

JEL classification : D92, Q30, Q40, Q42

Keywords : energy transition; non renewable resource; renewable energy; adjustment costs; capacity constraints. 


\section{Introduction}

The transition between different natural resources exploitation regimes typically takes time. While significantly in use since the sixteenth century in Great Britain, coal mining replaced only very slowly charcoal in iron processing or wood in energy provision until the nineteenth century (Wrigley, 2010, Fouquet, 2008). The same may be said for the use of oil and natural gas which developed over a sixty years range period since the end of the nineteenth century. More recently, the development of new energy sources like solar or biofuel is expected to extend well over the current century (Nakicenovic et al., 1998, chap 5). Most policy proposals to develop such alternatives in order to mitigate climate change are explicitly time dependent, the European Union 20-20-20 plan being one prominent example. Current and prospected energy policies thus strongly acknowledge the time lags implied by long run adaptations of the present energy mix. In some sense the climate challenge may be seen as a time to act problem, balancing the speed of possible adaptations to climate change with the speed of such a change.

This time to build issue covers many different problems ranging from the need of a sufficiently rapid technical progress to develop economically relevant energy alternatives to a sufficiently fast investment pace in natural resources services provision. Adaptation, or more generally development of the exploitation of natural resources is a costly process falling under the heading of 'adjustment costs' in investment economics. This issue of adjustment costs is not only of concern for the development of new resources but also for the development of existing ones, a well known feature of resource industries, either for the exploration and exploitation of new oil fields or for mineral resources.

The objectives of the paper are two-fold. First we want to stress the importance of investment constraints over the development of renewable energy alternatives. In order to focus upon the investment issue we shall dispense from considering explicitly the pollution problems raised by burning fossil fuels. Hence the main motivation for developing energy alternatives will be the increasing scarcity of non renewable fossil fuels like oil. For the same reason we shall not deal with the important issue of technical progress or learningby-doing in the use of new energy sources. This issue has raised significant 
attention in the macroeconomic endogenous growth literature recently (Acemoglu et al., 2011) but the precise micro foundations of this analysis, both at the firm level and at the energy sector level remain to be carefully settled. Technical progress should result into the generation of higher quality capital goods, an issue which would require to plug the analysis inside some vintage capital model, a study worth a dedicated research.

Second we want to explicitly consider the price implications of the development of renewable energy. One should expect that the gradual increase of renewable energy inside the energy mix will affect both the energy price trajectory and the depletion path of the already in use non renewable resource. Conversely, the time path of investment into renewable alternatives should depend upon the relative scarcity of the non renewable resource. Such possible linkages have attracted a lot of attention in the climate change regulation literature recently, under the heading of the so-called 'Green Paradox' dilemma.

To deal with this issue we depart both from the usual investment analysis at the individual firm level and from the aggregate studies at a macro level. We consider a partial equilibrium setting where the energy sector is composed of a population of identical competitive firms either producing energy from a non renewable resource or from a renewable one. Furthermore we assume that the renewable energy industry has to purchase specific equipments, linking at the equilibrium the dynamics of the energy price to the dynamics of the renewable energy capital input price. We assume an upward slopping supply curve of specific equipment of the renewable energy industry or equivalently an increasing marginal cost curve of equipment provision to the renewable industry. Thus the renewable industry faces external adjustment costs in the Lucas $\left(1967^{a}\right)$ sense rather than internal adjustment costs in the Gould (1968) sense. For simplicity we assume constant average and marginal variable operating costs in the non renewable and renewable energy industries and a lower operating cost of non renewable energy.

Adjustment costs have received a lot of attention in investment theory, seminal contributions to this literature being Lucas $\left(1967^{a, b}\right)$, Gould (1968) and Treadway (1969). However, while fully acknowledged as an important issue in natural resource development problems at least since 
Hotelling, (1931) $)^{1}$, it has attracted a relatively modest attention from resource economists. The textbook treatments of substitution between natural resources (for example Herfindahl and Kneese, 1974) do not consider explicitly adjustment costs. This results into a description of the history of natural resources use development as a sequence of time phases of exploitation of a dominant resource (the wood age, the coal age, the oil age) separated by quick transitions from a dominant resource to another one, according to their relative cost order.

It is a commonplace observation (IEA, 2013) that in energy production, natural resources rather coexist than override each other. The possibility of various types of capacity constraints in natural resources provision lies at the heart of the rare attempts of the literature to provide a more realistic account of the dynamics of energy transitions (Kemp and Long, 1980, Amigues et al., 1998, Holland, 2003).

In the resource literature, reference to adjustment costs has served two main purposes. The first one concerns the validity of the Hotelling rule. The rule predicts an increasing trend of the non renewable resources prices together with a decline of the production rate of these resources. However, the currently available data reveals that despite a formidable increase of the exploitation rate of the main mineral resources over the two last centuries, their prices have remained more or less constant in real terms, showing no definite upward trend that could be explained by the Hotelling rule (Gaudet, 2007). Various explanations, have been proposed to reconcile the theory with the data (Livernois, 2009). On the theoretical side, it has been stressed that capacity constraints, technical progress, dynamic costs structures, uncertainties or market imperfections may account for the observed resource price trends. On the empirical side, the difficulty to gather relevant data over long time periods and various econometric estimation issues can also explain the apparent lack of evidence concerning the Hotelling rule.

\footnotetext{
1 "The cases considered in the earlier part of this paper all led to solutions in which the rate of production of a mine always decreases. By considering the influence of fixed investments and the cost of accelerating production at the beginning, we may be led to production curves which rise continuously from zero to a maximum, and then fall more slowly as exhaustion approaches. Certain production curves of this type have been found statistically to exist for whole industries of the extractive type, such as petroleum production." Hotelling, 1931, p 164.
} 
In the context of the theory of the mine, the fact that investment costs may result in constant resource prices has been shown by Campbell (1980), extending the previous work of Puu (1977). The strength of the Campbell model is to take explicitly into account the consequences of extraction capacity constraints over the resource price, but its main weakness, as emphasized by Gaudet (1983), is to transform the gradual capacity development process into a static investment problem, the mining industry having to choose initially a given production capacity held constant over the whole mine life duration. The resource price consequences of the firms investment decisions have also been examined in a similar context by Lasserre (1986).

The second purpose is the study of transitions between different resources, main contributions to this issue being Olsen (1989) and Cairns and Lasserre (1991). The analysis of Olsen and Cairns and Lasserre are complex and focus upon the transition between different non renewable resources under adjustment costs.

Few parallel effort has been made to describe the transition between a non renewable resource and a renewable one, this last resource being submitted to adjustment costs in its productive capacity. One important contribution in this direction is Tsur and Zemel (2011) which model the capital accumulation process in producing solar energy under competition with existing fossil fuel resources. However Tsur and Zemel do not take into account the exhaustible nature of fossil fuels, assuming a forever constant supply of such resources.

The study of transitions between energy sources clearly appears useful in the context of the climate policy debate, the development of 'green' energy alternatives being a major topic in this respect. The perception that green energies develop at a too slow rate is commonplace in the public debate and it already exist several policy initiatives aimed at subsidizing renewable energy sources in industrialized countries. The rationale for such subsidies has been questioned recently in the so-called 'green paradox' debate (Sinn, 2012). This is the point raised in conjunction with capacity constraints by Gronwald, Long and Röpke (2013) in a recent work. The issue has also been studied by Smulders, Tsur and Zemel (2012) within the context of macro-economic growth theory, but without explicit consideration for the exhaustibility of the polluting resource. 
The works of Switzer and Salant (1983) and Powell and Oren (1989) are close to our work using a similar model. The same can be said of the Wirl (1991) work, although the Wirl contribution is cast in a dynamic imperfect competition setting where the incumbent non renewable resource extracting industry faces the potential entry of renewable energy producers submitted to production capacity development constraints. The findings of these authors are close to ours in several aspects. All claim that renewable energy should enter the market once the producers can at least cover their marginal production costs. Powell and Oren, as Wirl, show also the existence of an initial development phase where the industry builds some renewable energy capacity before actually using it. However we show that this outcome is only one possibility among the four possible energy transition scenarios we identify in our paper.

Our main findings are the following. With sufficient non renewable resource initial endowments, the equilibrium path is a sequence of three phases, a first phase during which only the cheaper non renewable resource is exploited, followed by a transition phase of simultaneous use of the non renewable and renewable energy sources up to some finite time when the non renewable resource reserves become exhausted. This transition phase is followed by a last renewable energy use phase of infinite duration. During the first and second phases, the energy price increases, following a Hotelling like path. It peaks at the depletion time of the non renewable resource and next decreases during the last phase because of the continuous expansion of the renewable energy production capacity which occurs all over this phase. This overall shape of the energy transition has been already identified by Powell and Oren or Wirl. However, all these authors posit a zero initial level of marginal investment cost, which implicitly assumes that the equipment industry could start supplying productive capacity with no inputs. We make instead the more realistic assumption of a strictly positive minimal price of equipment. This opens new energy transitions possibilities than the ones identified in this earlier literature.

The development of the renewable energy alternative may follow two possible scenarios. Under some conditions, the energy industry should start to invest into the renewable alternative before using it, waiting for the energy price to reach the variable average production cost level of renewable energy to produce. This is the equilibrium scenario with zero initial adjustment costs described by Wirl and others. In such scenarios, the initial renewable 
energy production capacity development and the beginning of renewable energy production happen in strict sequence. But, because of positive initial adjustment costs, it is also possible that the industry should delay the development of renewable energy only after a sufficient gap between the energy price and the marginal production cost of the alternative has been achieved. In this case capacity development and energy production will be synchronized during the transition.

We also show that the features of the energy transition may be in fact independent from the initial scarcity of the non renewable resource. In some scenarios, a higher availability of this resource simply translates farther in time the same renewable energy investment plan. This feature of the energy transition applies both in a scenario of early building of the production capacity or in a scenario of simultaneous building of the capacity together with the development of the production of renewable energy. However, if the price at which renewable energy production becomes competitive is independent from the non renewable resource scarcity in the first situation, this is not the case in the second one.

In some scenarios, the investment into renewable energy first rises and then begins to decrease strictly before the depletion of the non renewable resource, the other possibility being a constant decline of the investment rate after an initial jump up. In all scenarios, the industry incurs negative returns over its investments at the early stage of the transition. After the exhaustion of the non renewable resource, the renewable energy sector will continue to expand its production capacity up to some long run efficient production capacity level. This implies that it is never optimal for the renewable energy sector to hold this long run capacity level at the end of the transition phase.

Last, we show that the renewable energy production capacity at the time at which it replaces completely non renewable energy depends only of the characteristics of the last pure renewable energy production phase and not of the features of the energy transition. This applies also to the peak energy price at the time of exhaustion of the non renewable resource, or 'peak oil' price, which we prove rather counterintuitively to be independent not only of the renewable energy production capacity building process, but even of the scarcity of the non renewable resource itself. 
The paper is organized as follows. We describe in the next section a model of transition between a non renewable resource and a renewable resource facing capacity development constraints. Under our constant variable marginal costs assumption, it turns out that the non renewable resource will be exhausted in finite time. Thus we proceed in section 3 to the description of the ultimate phase of only renewable energy production. This last time phase may be described using the phase diagram technique developed by Treadway (1969). Section 4 examines the features of the transition phase between non renewable and renewable energy, focusing upon the description of the investment path into the expansion of renewable energy. Section 5 provides a closed form solution to the model and shows that the characteristics of the investment policy into renewable energy may be largely independent from the scarcity of the non renewable resource. Section 6 discusses the robustness of the results and their policy implications. Section 7 concludes.

\section{The model}

The economy has access to two different primary energy sources. The first one is a non renewable resource, say oil. Let us denote by $X(t)$ the available oil stock at time $t$, by $X_{0}$ its initial endowment, $X(0)=X_{0}$, and by $x(t)$ its instantaneous extraction rate, so that $\dot{X}(t)=-x(t)$. Assume a proportional normalized to one delivery of energy services from oil extraction so that $x(t)$ denotes also the energy services generation rate from oil exploitation. The provision of energy services from the oil primary source to the users incurs a constant unit and marginal cost $c_{x}$. We dispense from considering the possible pollution problems raised by burning oil to produce energy.

The second energy source is a renewable resource, let say solar, and denote by $y(t)$ the flow of energy services from the solar source. The delivery of such energy services incurs a constant unit and marginal cost $c_{y}$. The energy services delivered by the industry from any primary source are perfect substitutes for the users. Let $q(t)=x(t)+y(t)$ be the aggregate energy services supply by the energy sector. $p$ denotes the energy price and $p^{d}(q)$ is the inverse demand function, $p^{d}(q): R_{+} \rightarrow R_{+}$, is continuous and differentiable with $d p^{d}(q) / d q<0$ and $\lim _{q \downarrow 0} p^{d}(q)=+\infty$. Last, let us denote by $q^{d}(p)$ the direct energy demand function where $d q^{d}(p) / d p<0$. 
To use the solar source, the energy industry has to build a dedicated production capacity, say a solar panels stock. Maintaining the production capacity has a cost $c_{K}$ per unit of maintained capacity. The firms have thus to decide over a maintenance effort. Assume that any fraction of the capital stock which does not benefit from maintenance is definitively lost and that the scrapping costs are nil. Thus a negative adjustment of the capital stock is made possible by applying maintenance effort to only a fraction of the installed capacity. Let $K(t)$ be the installed and maintained solar production capacity at time $t$. There is no installed solar production capacity initially so that $K(0)=0$. Let $k(t)$ be the instantaneous purchase rate of solar equipment, $k(t) \geq 0$, and $\theta(t)$ the instantaneous proportional rate of scrapped equipment, so that the maintained capital stock dynamics is defined as $\dot{K}(t)=k(t)-\theta(t) K(t)$.

Each unit of maintained capacity is assumed to be able to deliver one unit of energy services thus: $y(t) \leq K(t)$. It will be shown that the production capacity constraint does not necessarily bind. More precisely, the industry can invest initially into capacity building without using it to produce solar energy.

The energy industry has to purchase its equipment over a specific market e.g. the solar panels market. Let $p_{K}(t)$ be the price of solar panels and denote by $k^{s}\left(p_{K}\right)$ the supply curve of solar panels. We assume that there exists some positive $p_{K}^{0}>0$ such that $k^{s}\left(p_{K}^{0}\right)=0$. We introduce this last feature for the sake of realism but it will appear that it allows for a much better understanding of the investment logic. Assume an increasing supply curve, that is $k^{s}(p):\left[p_{K}^{0}, \infty\right) \rightarrow R_{+}$is a continuous and differentiable function such that $d k^{s}\left(p_{K}\right) / d p_{k}>0$ and $k^{s}\left(p_{K}^{0}\right)=0$. The supply curve would identify to the marginal cost curve of the solar panel industry in a competitive situation.

Three remarks are in order at this stage. First, as pointed out in the introduction, investment theory with adjustment costs (e. g. Lucas, 1967-b) distinguishes between two kinds of costs: external costs associated to the purchase of new capital equipment and internal costs identified to specific costs of putting new equipment into a productive state together with the existing installations. The present formulation neglects these internal costs, the firms being able first, to run freely any level of available capacity at any time and second, to incorporate new equipment without incurring specific 
installation costs.

Secondly, it may be possible that $p_{K}(t)<c_{K}$. In such a case the industry should not apply any maintenance effort, scrap entirely the existing production capacity at time $t$ and next purchase a new one. Assume that $c_{K}<p_{K}^{0}$ to rule out such a possibility.

Thirdly, we are primarily interested in the logic of the energy transition between an exhaustible fossil fuel and a renewable alternative in a time to build context. To achieve this aim, we choose the simplest formulation of the production costs of energy services from the two sources. More complex forms, like increasing average costs functions or marginal cost functions depending on past cumulated extraction for example, would blur the analysis, since simultaneous exploitation of the two resources would become possible at the equilibrium even without any time to build issue. Adopting a constant marginal cost structure put the model inside the Herfindahl logic: without any capacity building problem, the two resources should be exploited in strict sequence, beginning with the least cost resource. The existence of a non trivial transition between the two energy sources will thus stand as a salient feature of the present framework. However, it should be expected that the precise characteristics of the transition are highly dependent upon the shape of the energy demand function and the equipment supply function. To achieve some degree of generality of the study, we retain for these functions only reasonable qualitative properties: a decreasing energy demand function and an increasing equipment supply function.

In the context of capacity investment costs, the both cases of a cheaper solar energy with respect to oil or a cheaper oil than solar appear to be worth a study. We shall concentrate upon the case of a cheaper oil energy that is assume: $c_{x}<c_{y}$. Thus absent any depletion of the oil resource, there should be no development of solar energy. It is the pure logic of resource exhaustion that will motivate the expansion of the solar energy alternative.

The energy industry is composed of competitive firms having access to the same technologies for energy services provision. Hence it does not matter to assign specialization into oil or solar energy generation to a given firm. Facing the same energy and solar equipment markets conditions, the firms should take identical decisions regarding output and inputs purchase poli- 
cies. At the equilibrium, their solar energy investment policy will be affected simultaneously by the present levels and future dynamics of the equipment price and the energy price. The supply curve of solar equipments being upward sloping, an increased speed of equipment accumulation should induce price increases upon the equipment market. Hence the market behavior will mimic at the equilibrium the features of the convex cost structure one finds in the standard investment models with internal adjustment costs.

Assuming perfect competition over both the energy and solar equipment markets, the energy sector has to design supply plans $\{(x(t), y(t)), t \geq$ $0\}$ and solar capacity investment and scrapping and/or maintaining plans $\{(\theta(t), k(t)), t \geq 0\}$ maximizing the sum of discounted profits. Let $r$ be the interest rate assumed to be constant. Formally the energy sector solves:

$$
\begin{aligned}
\max _{x(t), y(t), \theta(t), k(t)} & \int_{0}^{\infty}\left\{p(t)[x(t)+y(t)]-c_{x} x(t)-c_{y} y(t)\right. \\
& \left.-p_{K}(t) k(t)-c_{K} K(t)\right\} e^{-r t} d t \\
\text { s.t. } \quad & \dot{X}(t)=-x(t), X(0)=X_{0}>0 \text { given, } X(t) \geq 0 \\
& \dot{K}(t)=k(t)-\theta(t) K(t), K(0)=0 \text { given }, K(t) \geq 0 \\
& \theta(t) \geq 0, k(t) \geq 0, x(t) \geq 0, y(t) \geq 0, K(t)-y(t) \geq 0 .
\end{aligned}
$$

The current value Lagrangian of this problem writes (dropping the time argument for the ease of reading):

$$
\begin{aligned}
\mathcal{L}= & p(x+y)-c_{x} x-c_{y} y-p_{K} k-c_{K} K-\lambda_{X} x+\lambda_{K}(k-\theta K) \\
& +\gamma_{\theta} \theta+\gamma_{k} k+\gamma_{x} x+\gamma_{y} y+\nu_{K} K+\nu_{X} X+\gamma_{K}(K-y) .
\end{aligned}
$$

The Lagrangian maximization yields:

$$
\begin{aligned}
\mathcal{L}_{x}=0 & \Longrightarrow p=c_{x}+\lambda_{X}-\gamma_{x} \\
\mathcal{L}_{y}=0 & \Longrightarrow p=c_{y}+\gamma_{K}-\gamma_{y} \\
\mathcal{L}_{k}=0 & \Longrightarrow p_{K}=\lambda_{K}-\gamma_{k} \\
\mathcal{L}_{\theta}=0 & \Longrightarrow \lambda_{K} K=\gamma_{\theta}
\end{aligned}
$$

together with the usual complementary slackness conditions. The costate variables $\lambda_{X}$ and $\lambda_{K}$ must satisfy, when differentiable:

$$
\dot{\lambda}_{X}=r \lambda_{X}-\mathcal{L}_{X} \Longrightarrow \dot{\lambda}_{X}=r \lambda_{X}-\nu_{X}
$$


and:

$$
\dot{\lambda}_{K}=r \lambda_{K}-\mathcal{L}_{K} \Longrightarrow \dot{\lambda}_{K}=(r+\theta) \lambda_{K}+c_{K}-\gamma_{K}-\nu_{K},
$$

with $\nu_{X} \geq 0(=0$ if $X>0)$ and $\nu_{K} \geq 0(=0$ if $K>0)$.

The transversality condition at infinity reads:

$$
\lim _{t \uparrow \infty}\left[\lambda_{X}(t) X(t)+\lambda_{K}(t) K(t)\right] e^{-r t}=0
$$

Let $t_{X}$ be the time at which oil is exhausted, then (2.5) gives the form of the Hotelling rule when the average costs are constant:

$$
\lambda_{X}(t)=\lambda_{X} e^{r t} \text { where } \lambda_{X} \equiv \lambda_{X}(0), t \leq t_{X} .
$$

In the above system, the multiplier $\gamma_{K}$ is the shadow marginal value of the solar production capacity $K$. During any time period of solar energy production, $\gamma_{y}=0$ since $y>0$ and, according to (2.2): $\gamma_{K}=p-c_{y}$. Thus $\gamma_{K}$ is the gross unit margin in the solar energy activity, from which we must deduce the unit maintenance cost, $c_{K}$, to get the net margin, or operative cost, we denote by $\beta_{K}$ : $\beta_{K} \equiv \gamma_{K}-c_{K}$.

Consider the condition (2.4) determining the scrapping rate, equivalently the maintenance rate, of the installed solar production capacity $K$. Assume that $\theta>0$, hence $\gamma_{\theta}=0$, so that $\lambda_{K} K=0$. Thus either $\lambda_{K}=0$, or $K=0$ or both. When $K=0$, necessarily $\theta=0$ and we could be left with $\lambda_{K}=0$ during a period within which the firms would hold a positive solar production capacity $K>0$. Rather than incurring a marginal cost $c_{K}>0$ for maintaining a potentially productive solar energy capital $K>0$, the value of which is nil, it would be a more profitable management policy to scrap it completely at zero cost. This argument holds for any $\theta>0$, however large, and any $K>0$, however small. We conclude that a profit maximizing industry should never apply at any time $t$ a positive scrapping rate, $\theta(t)>0$, to the installed capital $K(t)$ at this time. ${ }^{2}$

At any time $t$, the shadow marginal value of the solar production capital, $\lambda_{K}(t)$, must be equal to the sum of the future discounted net margins, that

\footnotetext{
${ }^{2}$ Clearly the argument would not hold under non stationary surplus functions, for example a marginal surplus function first increasing through time and next decreasing.
} 
is ${ }^{3}$ :

$$
\lambda_{K}(t)=\int_{t}^{\infty} \beta_{K}(\tau) e^{-r(\tau-t)} d \tau .
$$

The above relation shows that it identifies with the cumulated present value of the opportunity cost of the capacity constraint over solar energy generation net of the maintenance cost of capital.

Then, the condition (2.3) states that at the equilibrium, when the energy industry invests in solar capacity, $k>0$, the price of the solar equipment $p_{K}$ must be equal to the shadow marginal value of this capital:

$$
p_{K}(t)=\lambda_{K}(t)=\int_{t}^{\infty}\left(p(\tau)-\left(c_{K}+c_{y}\right)\right) e^{-r(\tau-t)} d \tau
$$

Alternatively, the condition (2.10) may be seen as the no pure profit condition that must prevail in a competitive situation.

Before turning towards a detailed analysis of the implications of the necessary conditions, let us sketch a reasonable guess solution to the problem.

(i) Since $c_{x}<c_{y}$ and solar development has to be started from scratch, the non renewable resource will be put into exploitation right from the beginning.

(ii) There cannot be an abrupt transition from oil to solar energy as in the textbook Herfindahl model, since solar capacity building is costly and the supply curve of solar equipment has been assumed to be upward slopping. Hence, unlike the Herfindahl model, there should exist a phase of simultaneous exploitation of both solar and oil energy, despite the fact that their respective average costs are constant and that $c_{x}<c_{y}$ by assumption.

(iii) Depending upon the cost advantage of oil with respect to solar and the other features of the model, it may or not be the case that the investment into solar production capacity building will be delayed in time, opening the door for a first phase of only oil exploitation before the transition towards solar energy.

\footnotetext{
${ }^{3}$ Note that (2.9) holds provided that the transversality condition (2.7) be satisfied.
} 
(iv) In this last case, it appears possible that the industry should invest into solar production capacity before using it to produce renewable energy.

(v) Since the marginal cost of oil energy services has been assumed to be constant, oil should be depleted in finite time.

(vi) After oil depletion, only the solar energy sector will remain active. Depending upon the previously accumulated production capacity, it may or not be the case that capacity will continue to expand. In the first case, energy supply should increase while the energy price should decline over time.

That the non renewable resource should be exhausted in finite time results from the following argument. Assume to the contrary that the resource is exhausted only asymptotically. Then the Hotelling rule expressed in (2.8) implies that the energy price should increase up to infinity in infinite time. But in this case there should exist some finite time, $\underline{t}$, when an investment into solar energy generation becomes profitable, that is when the energy price is sufficiently high to cover at least the minimal complete marginal cost $r p_{K}^{0}+c_{K}+c_{y}$ of solar energy. Since the energy price should continue to grow over time after $\underline{t}$, the industry will accumulate solar production capacities. To any finite capacity so accumulated, $K$, there exists a finite price level $p=p^{d}(K)$ such that solar energy could serve the whole energy demand at this price level, hence a contradiction.

The above features suggest the following study plan. We describe in the next section the last phase of only solar energy use. Next we characterize the properties of the transition phase from oil toward solar energy. Different possible energy transition scenarios emerge from this study. We thus have to identify the domain of validity of these scenarios. This is achieved by means of an algorithmic procedure, providing a closed form solution to the present model. 


\section{The pure solar phase}

After $t_{X}$, the oil exhaustion time, solar is the only primary source and the industry inherits a previously accumulated capacity $K\left(t_{X}\right)=K_{X}$. In this section we determine the perfect foresight equilibrium as a function of $K_{X}$, $K(t)$ being the only state variable of the system since $X(t)=0, t \geq t_{X}$.

\subsection{Limits within which the equilibrium is to stay at $K_{X}$}

To determine the limits within which $K_{X}$ must lie in order that $\{K(t)=$ $\left.K_{X}, p(t)=p^{d}\left(K_{X}\right), t \geq t_{X}\right\}$ be a perfect foresight equilibrium we must check why for the given future price path $\left\{p(t)=p^{d}\left(K_{X}\right), t \geq t_{X}\right\}$ the industry does neither increase nor decrease its inherited capacity, that is why $\left\{y(t)=K_{X}, t \geq t_{X}\right\}$ is on its supply path.

A first condition is that the industry be not in excess supply. If $p^{d}\left(K_{X}\right)<$ $c_{K}+c_{y}$, then at this price the current receipts are lower than the current costs. The energy industry is in excess capacity. Since $p^{d}(K)$ is a decreasing function of $K$, this level $\bar{K}$ solution of $p^{d}(K)=c_{K}+c_{y}$ is the lower bound of $K_{X}$ for which the industry is in excess capacity.

The second condition is that the industry be not in excess demand. Assume that there is no capacity investment after $t_{X}$. A slight increase of the capacity above $K_{X}$ would generate a marginal profit, in value at any time $t \geq t_{X}$ at which it is undergone, $v\left(K_{X}\right)$ :

$$
v\left(K_{X}\right)=\int_{t_{X}}^{\infty}\left[p^{d}\left(K_{X}\right)-c_{y}-c_{K}\right] e^{-r\left(t-t_{X}\right)} d t=\frac{p^{d}\left(K_{X}\right)-c_{y}-c_{K}}{r} .
$$

Assume that $p_{K}^{0}<v\left(K_{X}\right)$, then a slight investment $k>0$ at time $t$ would bear a cost $p_{K}^{0} k$ and generate a larger profit $v\left(K_{X}\right) k$. Thus a condition for not to be in excess demand is that $p_{K}^{0} \geq v\left(K_{X}\right)$.

Let $\hat{K}$ be the solution of $v\left(K_{X}\right)=p_{K}^{0}$, so that $p^{d}(\hat{K})-c_{y}=r p_{K}^{0}+c_{K}$. The term $r p_{K}^{0}$ is the current rental price of equipment purchased at price $p_{K}^{0}$ 
and $c_{K}$ is its current maintenance cost. Thus $r p_{K}^{0}+c_{K}$ is the current full marginal cost of a small investment while $p^{d}(\hat{K})-c_{y}$ is its current marginal benefit at the constant price path $\left\{p(t)=p^{d}(\hat{K}), t \geq t_{X}\right\}$.

Because $p^{d}(K)$ is a decreasing function of $K$, then $\hat{K}<\bar{K}$. For any inherited capacity $K_{X}$ within the above two benchmarks, $\hat{K}<K_{X}<\bar{K}$, if the industry foresights a price path $p(t)=p^{d}\left(K_{X}\right), t \geq t_{X}$ and stays at $K(t)=K_{X}$, then it is neither in excess supply nor in excess demand. Hence $\left\{p(t)=p^{d}\left(K_{X}\right), K(t)=K_{X}, t \geq t_{X}\right\}$ is a perfect foresight equilibrium.

\section{2 $\quad$ Starting from outside the limits}

Assume that $K_{X}>\bar{K}$. Then clearly the perfect foresight equilibrium is: $p(t)=p^{d}(\bar{K}), t \geq t_{X}$, the capacity being reduced to $\bar{K}$ by scraping immediately the excess capacity $K_{X}-\bar{K}$ at time $t_{X} \cdot{ }^{4}$

Assume now that the industry would be in excess demand by staying at $K_{X}$, that is $K_{X}<\hat{K}$. The problem is to find the price path of energy, $p(t)$, the price path of equipment, $p_{K}(t)$, and the investment path, $k(t)$, maximizing the profits of the energy industry and the equipment industry. This is equivalent to determine the solution of the following system of equations (3.1)-(3.2):

$$
\begin{aligned}
\dot{K}(t) & =k^{s}\left(\lambda_{K}(t)\right) \\
\dot{\lambda}_{K}(t) & =r \lambda_{K}(t)+c_{K}-\left(p^{d}(K(t))-c_{y}\right) .
\end{aligned}
$$

where $\lambda_{K}(t)=p_{K}(t)$, is the equipment price (c.f. (2.3) with $k>0$ ).

Equation (3.1) states that the capital investment $\dot{K}(t)=k(t)$, the equipment demand, is equal to the equipment supply of the equipment industry, $k^{s}\left(\lambda_{K}(t)\right)=k^{s}\left(p_{K}(t)\right)$. Equation (3.2) is the profit maximization condition of the capital accumulation plan of the energy industry for $p(t)=p^{d}(K(t))$, the foresighted equilibrium energy price. Remember that $\gamma_{K}=p-c_{y}$, that is $p^{d}(K(t))-c_{y}$ at the equilibrium while $\nu_{K}=0$ since $K>0$, hence the form (3.2) of (2.6).

\footnotetext{
${ }^{4}$ This is an example of an impulse decision. As shown later, it disappears along the perfect foresight equilibrium starting from $K(0)=0$ at $t=0$.
} 
The system (3.1)-(3.2) is a simple non linear differential system which can be studied with the phase diagram technique. The phase diagram in the $\left(K, \lambda_{K}\right)$ plane is pictured on Figure 1 (see Appendix A.1 for the details of the construction).

\section{Figure 1 about here}

We show in Appendix A.1 that the saddle branch graphed as a solid bold line is the only equilibrium solar capacity expansion path starting from $K_{X}<\hat{K}$. We denote by $\lambda_{K}^{X}(K)$ the equation of the saddle branch. Note that $d \lambda_{K}^{X}(K) / d K<0$ within the interval $\left[K_{X}, \hat{K}\right]$. In infinite time, the equilibrium path converges toward $\left(\hat{K}, p_{K}^{0}\right)$. The long run level of the solar energy generation capacity, hence the long run solar energy production rate, is the solution of $p(K)=c_{y}+c_{K}+r p_{K}^{0}$. Thus even in the very long run the gross marginal surplus from solar energy consumption will be higher than $c_{y}$, the marginal cost of solar production. Only in a case where the minimal marginal adjustment cost $p_{K}^{0}$ would be zero together with the maintenance costs, $c_{K}$, the equalization in the long run of the gross marginal surplus to the variable marginal cost $c_{y}$ would be profit maximizing for the solar industry.

Observe also that since $\lambda_{K}(t)=p_{K}(t)$ decreases along the saddle branch through (2.3), $k(t)=k^{s}\left(p_{K}(t)\right)$ also decreases down to zero. Investment into capacity building follows a smooth decreasing pattern toward zero. The production capacity of solar energy, hence the solar energy production rate, expands while the energy price decreases. We may conclude as follows:

Proposition P. 1 Consider the perfect foresight equilibrium following the exhaustion of the oil reserves at time $t_{X}$ starting with a previously accumulated solar capacity $K_{X}$. Let $\bar{K}$, the solution of $p^{d}(K)=c_{y}+c_{K}$, be the lower bound for which the industry would be in excess capacity and $\hat{K}$, the solution of $p^{d}(K)=c_{y}+c_{K}+r p_{K}^{0}$, be the upper bound for which the industry would be in excess demand for capacity. Clearly, $\hat{K}<\bar{K}$ and:

1. If $\hat{K}<K_{X}<\bar{K}$, then staying at $K_{X}$ for $t \geq t_{X}$ is the only perfect foresight equilibrium on the industry production capacity market. 
2. If $K_{X}>\bar{K}$, the equilibrium capacity is $\bar{K}$ and the excess capacity $K_{X}-\bar{K}$ is scraped immediately at time $t_{X}$.

3. If $K_{X}<\hat{K}$, the solar energy sector should permanently expand its production capacity $K(t)$ towards $\hat{K}$, a level attained only asymptotically in infinite time.

- During this capacity expansion phase, the investment level $k(t)$ into new solar equipment permanently decreases, converging down to zero in the very long run.

- The equilibrium price of solar equipment, $p_{K}(t)$, decreases and converges towards $p_{K}^{0}$.

- The production of solar energy is given by $y(t)=K(t)$, the capacity constraint being binding all along the time phase. Solar energy generation increases permanently towards $\hat{y}=\hat{K}$. The energy price decreases and converges down to $\hat{p} \equiv r p_{K}^{0}+c_{K}+c_{y}>c_{y}$. $y(t)$ increases at a decreasing rate while $p(t)$ decreases also at a decreasing rate.

The last phase of only solar energy use is a good illustration of the investment logic under adjustment costs in the line of Treadway. At each time when the energy supply capacity is increased, the equilibrium market reaction to this extra supply is an energy price decrease. This lowers the profitability prospects for future investments into capacity expansion. The result is a slowing trend of investment into solar energy generation equipment. The demand reaction effect is partly counterbalanced by the decreasing returns to scale over equipment provision. By reducing progressively the purchase of new capital, the energy industry spans downward the supply curve, that is the marginal cost curve of the solar panels industry. The resulting equipment cost cut down restores partially the loss of profitability induced by the demand reaction to the solar production capacity expansion.

As will be shown below, the argument extends to the capacity accumulation policy before the depletion of the non renewable resource. The above driving demand and cost forces explain also why the energy industry should not end at the highest capacity economically profitable when the oil resource becomes exhausted, that is $K_{X}<\hat{K}$. 


\section{Transition toward the pure solar economy}

Two features strongly shape the equilibrium transition toward the pure solar economy. The first one is that before $t_{X}$, the economy consumes the oil resource. Hence the energy price increases according to the Hotelling rule: $p(t)=c_{x}+\lambda_{X} e^{r t}$ (c.f. (2.1)). The second feature is that convex adjustments costs prevent an instantaneous transition from a pure oil economy to a pure solar one. Hence, before $t_{X}$ there must exists a phase of joint exploitation of the two resources and a capital building phase at least as large since producing solar energy requires capital.

\subsection{Equilibrium constraint on the solar energy produc- tion capital at the oil depletion time}

The Hotelling rule before $t_{X}$ and the characteristics of the equilibrium energy price path after $t_{X}$, stated in Proposition 1, together imply that $K_{X}<\hat{K}$.

Assume to the contrary that $K_{X} \geq \hat{K}$. From Proposition 1, after $t_{X}$ the energy price is constant and equal to $p(t)=p^{d}\left(\min \left\{K_{X}, \bar{K}\right\}\right), t>t_{X}$. This price does not cover the current full marginal cost $r p_{K}+c_{K}+c_{y}>r p_{K}^{0}+c_{K}+c_{y}$ of any piece of equipment purchased before $t_{X}$ at a price $p_{K}>p_{K}^{0}$ when $k>0$. Since the equilibrium energy price path must be continuous at time $t_{X}$, then $p(t)<p^{d}\left(\min \left\{K_{X}, \bar{K}\right\}\right)$ before $t_{X}$ thanks to the Hotelling rule. Thus the full marginal cost is never recovered, contradicting profit maximization by the energy industry.

For $K_{X}<\hat{K}$, the equilibrium paths of $\lambda_{K}(t)$ and $K(t)$ must move along the stable branch $\lambda_{K}^{X}(K)$ of the phase plane illustrated in Figure 1. Given that the equilibrium paths of $\lambda_{K}$ and $p$ must be continuous at time $t_{X}$, then the equilibrium values $\lambda_{K}\left(t_{X}\right)$ and $p\left(t_{X}\right)$ must be equal respectively to $\lambda_{K}^{X}\left(K_{X}\right)$ and $p^{d}\left(K_{X}\right)$, the mining rent $\lambda_{X} e^{r t_{X}}$ being thus equal to $p^{d}\left(K_{X}\right)-c_{x}$. Note that $\lambda_{K}^{X}(K)$ being a decreasing function of $K$ and $K(t)$ being increasing over time after $t_{X}, \dot{\lambda}_{K}\left(t_{X}^{+}\right)<0 .{ }^{5}$

\footnotetext{
${ }^{5}$ From now on we denote by $h\left(t^{-}\right) \equiv \lim _{\tau \uparrow t} h(\tau)$ and $h\left(t^{+}\right) \equiv \lim _{\tau \downarrow t} h(\tau)$ respectively
} 
Last, the continuity of the energy price path and of the solar capital path at $t_{X}$ together imply that the oil consumption smoothly vanishes at $t_{X}$, $x\left(t_{X}^{-}\right)=0$, since $q\left(t_{X}^{+}\right)=y\left(t_{X}^{+}\right)=K_{X}$ and $p\left(t_{X}^{-}\right)=p\left(t_{X}^{+}\right)=p^{d}\left(K_{X}\right)$. The following Proposition summarizes these findings.

Proposition P. 2 Along the equilibrium path, at the oil exhaustion time $t_{X}$ : $K_{X}<\hat{K}, \lambda_{K}\left(t_{X}\right)=\lambda_{K}^{X}\left(K_{X}\right), p\left(t_{X}\right)=p^{d}\left(K_{X}\right), \lambda_{X} e^{r t_{X}}=p^{d}\left(K_{X}\right)-c_{x}$ and $x\left(t_{X}^{-}\right)=0$.

The following immediate implication of Proposition 2 and of the dynamics of $\lambda_{K}(t)$ before $t_{X}$ resulting from (2.6) will appear very useful for characterizing the equilibrium investment path in the paragraph 4.2.2 (see Appendix A.2 for a formal proof).

Corollary $1 \lambda_{K}(t)$ is time differentiable at $t_{X}$ and $\dot{\lambda}_{K}\left(t_{X}^{-}\right)=\dot{\lambda}_{K}\left(t_{X}^{+}\right)<0$.

Note that, being endogenously determined, $t_{X}$ and $K_{X}$ link what happens before and after the exhaustion of oil at the equilibrium.

\subsection{Main characteristics of the energy transition}

Let $t_{K}$ be the time at which the industry begins to accumulate solar energy production capital and $t_{y}$ be the time at which the industry begins to produce solar energy, $0 \leq t_{K} \leq t_{y}$.

\subsubsection{Beginning to accumulate solar capacity and beginning to produce solar energy}

It cannot be a priori excluded that the industry accumulates capital, $K$, before producing solar energy, that is $t_{K}<t_{y}$. Clearly, for energy price levels the left and right end limits at $t$ of any time function $h(t)$, when these limits exist. 
such that $p(t)<c_{y}$, the solar energy production rate must be nil (c.f. (2.2)), these price levels not covering the operative cost. But this does not imply that the industry should not accumulate any solar capacity before that time at which $p(t)=c_{y}$ and solar energy production could begin.

Assume that the energy firm faces some given increasing price schedule, $p(t)$, before $t_{X}$ (this will be the case at the equilibrium). Let $\bar{t}_{y}$ be the time at which $p(t)=c_{y}$, not necessarily equal to $t_{y}$. The firm can thus cover the variable cost from $\bar{t}_{y}$ onward. If it decides to produce solar energy at time $\bar{t}_{y}$, some capacity is needed. For the firm to hold capacity at time $\bar{t}_{y}$, it is necessary that:

$$
\lambda_{K}\left(\bar{t}_{y}\right)=\int_{\bar{t}_{y}}^{\infty}\left[p(t)-c_{y}-c_{K}\right] e^{-r\left(t-\bar{t}_{y}\right)} d t \geq p_{K}^{0}
$$

for the given price schedule $p(t)$. It is immediately verified that:

$$
\dot{\lambda}_{K}\left(\bar{t}_{y}\right)=r \lambda_{K}\left(\bar{t}_{y}\right)-\left[p\left(\bar{t}_{y}\right)-c_{y}-c_{K}\right]=r \lambda_{K}\left(\bar{t}_{y}\right)+c_{K} \geq r p_{K}^{0}+c_{K}>0 .
$$

Thus $\lambda_{K}(t)$ increases over time around $\bar{t}_{y}$. Furthermore, $p(t)$ being increasing before $\bar{t}_{y}, \lambda_{K}(t)$ increases also before $\bar{t}_{y}$. Then, depending on the level of $p_{K}^{0}$, the firm can be in three situations:

(i) If $\lambda_{K}\left(\bar{t}_{y}\right)>p_{K}^{0}$, there exists a unique $t_{K}, t_{K}<\bar{t}_{y}$, such that $\lambda_{K}\left(t_{K}\right)=$ $p_{K}^{0}$. This means that the firm should have begun to invest strictly before $\bar{t}_{y}$. But since $p(t)<c_{y}$ within the time interval $\left[t_{K}, \bar{t}_{y}\right)$, the firm should continuously invest without producing solar energy.

(ii) In the case $\lambda_{K}\left(\bar{t}_{y}\right)=p_{K}^{0}$, the firm begins to simultaneously invest and produce solar energy from $\bar{t}_{y}$ onward.

(iii) If $\lambda_{K}\left(\bar{t}_{y}\right)<p_{K}^{0}$, the energy price is sufficiently high to cover the variable cost but the minimal investment cost is too high to justify investing in solar production capacity. Thus the firm waits until the energy price is sufficiently high for $\lambda_{K}(t)=p_{K}^{0}$. At this time, the firm starts simultaneously to invest and produce solar energy.

Remark that if $p_{K}^{0}=0$ and $c_{K}=0$, only the first possibility remains, meaning that the industry always invests in capacity building before starting to 
produce renewable energy. Note also the crucial role of the time increasing pattern of the energy price. Facing for example a flat price level, $\bar{p}$, either $\bar{p}<c_{y}$ and the firm never invest or produce solar energy, either $\bar{p}>c_{y}$ and the firm starts immediately to invest and produce from the competitive solar source.

It is thus possible that the industry begins to develop the solar alternative before using it to produce energy. This is a common feature of R\&D models where the industry has to invest into costly research efforts in order to attain a sufficiently productive technological stage. There is no explicit R\&D process in this model, and the possibility of a delay between the beginning of the investment plan and the beginning of the production plan of solar energy appears here as an implication of the Hotelling rule constantly driving up the equilibrium energy price.

This possibility is also strongly tied to the increasing pattern of the solar equipment supply function $k^{s}\left(p_{K}\right)$, the levels of the minimal unitary capital cost, $p_{K}^{0}$, the maintenance cost, $c_{K}$, and the interest rate, $r$. For a low minimal cost, $p_{K}^{0}$, a supply function initially flat over a small price range and next poorly elastic, low maintenance costs, $c_{K}$ and interest rate, $r$, it may happen that accumulating capital before $t_{y}$ minimizes the building cost of $K\left(t_{y}\right)$. However, delaying the production of solar energy although having production capital at disposal is only possible when the variable cost $c_{y}$ is positive and sufficiently high, or alternatively if initially the oil energy price, $c_{x}+\lambda_{X} e^{r t}$, is sufficiently low.

From the Proposition 2 and the above developments, we conclude that there may exist four and only four equilibrium scenarios before oil depletion.

The first two types of scenarios begin with an initial phase of only oil exploitation and a smooth start of the investment policy:

- Scenario 1 is a three phases scenario where $0 \leq t_{K}<t_{y}<t_{X}$ and $k\left(t_{K}^{+}\right)=0$.

- Scenario 2 is a two phases scenario where $0 \leq t_{K}=t_{y}<t_{X}$ and $k\left(t_{K}^{+}\right)=0$. 
In the two other scenarios, solar production capital building occurs immediately at a strictly positive rate because of oil scarcity:

- Scenario 3 is a two phases scenario where $0=t_{K}<t_{y}<t_{X}$ and $k\left(t_{K}\right)>0$.

- Scenario 4 is a one phase scenario where $0=t_{K}=t_{y}<t_{X}$ and $k\left(t_{K}\right)>0$.

Scenarios where $t_{y}=t_{X}$ are excluded by the Proposition 2. Since at $t_{X}$, $p\left(t_{X}\right)>c_{K}+c_{y}$, then there must exist a time interval $\left(t_{X}-\delta, t_{X}^{-}\right), \delta>0$, during which $p(t)>c_{y}$ and hence $y(t)=K(t)>0$.

Before proceeding to the description of the solar energy investment plan in the different scenarios, let us sketch the main features of the energy price and quantity dynamics during the energy transition. The energy price, $p(t)$, permanently increases implying that the total energy consumption, $q(t)$ should decline over time. Once solar energy is introduced, the progressive accumulation of production capacity induces an increased use of solar energy inside the energy mix, oil consumption decreasing at a higher rate than total energy consumption. These features apply in all scenarios. Note that in the scenarios 1 and 3 of a delayed introduction of solar energy with respect to the solar panels investment plan, that is when $t_{K}<t_{y}$, the consumption of solar energy jumps up from zero to the available capacity level at time $t_{y}$, $y\left(t_{y}^{+}\right)=K\left(t_{y}\right)$. Oil consumption makes a parallel downward jump at $t_{y}$, total energy consumption having to be time continuous.

\subsubsection{Equilibrium investment dynamics in solar energy genera- tion}

Assume first that the oil endowment $X_{0}$ is large so that $\lambda_{X}$ is small and there exists an initial phase during which $c_{x}+\lambda_{X} e^{r t}<c_{y}$. Hence, only oil is exploited initially and the possible scenarios are of type 1 or 2 .

Consider the scenarios of type 1 where $0<t_{K}<t_{y}<t_{X}$. During the 
phase $\left(t_{K}, t_{y}\right)$ of capacity investment without solar energy production, the dynamics of $\lambda_{K}(t)$ satisfies:

$$
\lambda_{K}\left(t_{K}\right)=p_{K}^{0} \quad \text { and } \quad \dot{\lambda}_{K}(t)=r \lambda_{K}(t)+c_{K}=r p_{K}(t)+c_{K} .
$$

The first equality means that the capital accumulation process starts smoothly: $k\left(t_{K}^{+}\right)=0$. The reason is simple. Assume that $k\left(t_{K}^{+}\right)>0$, hence $p_{K}\left(t_{K}^{+}\right)>p_{K}^{0}$. Then reducing slightly the investment at $t_{K}^{+}$by $d k$ and starting to invest slightly earlier at $t_{K}^{-}$would allow for an investment cost reduction approximatively equal to $\left[p_{K}\left(t_{K}^{+}\right)-p_{K}^{0}\right] d k$. The second equality means that the installed capital is not scraped because its value increases sufficiently to cover its rental price, $r \lambda_{K}$, and its maintenance cost, $c_{K}$, but no more.

At $t_{y}, p\left(t_{y}\right)=c_{y}$ and the next phase is a phase of joint use of both resources during which the dynamics of $\lambda_{K}(t)$ is given by:

$$
\dot{\lambda}_{K}(t)=r \lambda_{K}(t)+c_{K}-\left(p(t)-c_{y}\right)=r p_{K}(t)+c_{K}-\left(c_{x}+\lambda_{X} e^{r t}-c_{y}\right) .
$$

Note that a $t_{y}, \dot{\lambda}_{K}(t)$ is well defined but the second order time derivative $\ddot{\lambda}_{K}(t)$ jumps downward by $r \lambda_{X} e^{r t}$, a point we check in appendix A.2. Hence after $t_{y}$, the increase of $\lambda_{K}(t)$ begins to decelerate.

The maximum of $\lambda_{K}(t)$ is attained a the time $\bar{t}$ at which $\dot{\lambda}_{K}(t)=0$, that is $r \lambda_{K}(t)+c_{K}=c_{x}+\lambda_{X} e^{r t}-c_{y}$, equivalently the time at which $r \lambda_{K}(t)=\beta_{K}(t)$, (see Figure 2). At time $\bar{t}, \dot{\lambda}_{K}(\bar{t})=0$ implies that:

$$
\ddot{\lambda}_{K}(\bar{t})=r \dot{\lambda}_{K}(\bar{t})-r \lambda_{X} e^{r \bar{t}}=-r \lambda_{X} e^{r \bar{t}}<0,
$$

thus the maximum is unique. Appendix A.2 checks that $\bar{t}<t_{X}$.

The Figure 2 illustrates the investment dynamics into solar capacity in the scenario 1 in terms of the dynamics of the dual variables $\lambda_{K}$ and $\beta_{K}$, where:

$$
\beta_{K}(t)= \begin{cases}-c_{K} / r & , t<t_{y} \\ c_{x}+\lambda_{X} e^{r t}-\left(c_{K}+c_{y}\right) & , t_{y} \leq t \leq t_{X} \\ p^{d}(K(t))-\left(c_{K}+c_{y}\right) & , t_{X}<t .\end{cases}
$$

Figure 2 about here 
The Figure 2 shows that the cash flow balance from one piece of equipment may have quite complex evolutions, alternating negative returns periods and positive ones. For equipments purchased between $t_{K}$ and $\bar{t}$, like those purchased at time $t_{1}^{*}$ on Figure 2, the purchase price is $\lambda_{K}\left(t_{1}^{*}\right)=p_{K}\left(t_{1}^{*}\right) \equiv p_{K}^{*}$. The net cash flow dynamics is a three phases sequence. During a first phase $\left(t_{1}^{*}, t_{2}^{*}\right), r \lambda_{K}\left(t_{1}^{*}\right)=r p_{K}^{*}>\beta_{K}(t)$ shows that the revenue is lower than the rental cost. During a second phase $\left(t_{2}^{*}, t_{3}^{*}\right)$, the reverse holds and during the third phase, $\left(t_{3}^{*}, \infty\right)$, the revenue is again lower than the rental cost. Thus, for pieces of equipment purchased at time $t_{1}^{*}$, the industry experiences first negative returns, then positive returns and last negative returns once again. For pieces of equipment purchased after $\bar{t}$, the cash flow balance is a sequence of two phases, a first phase of positive returns followed by an infinite duration phase of negative returns.

During the first pure investment phase, the net opportunity cost of the capacity constraint, $\beta_{K}$, remains at its minimal constant level $-c_{K}$. $\beta_{K}$ grows over time once solar energy is introduced within the energy mix. Thus investing early allows to reduce the cost of the capacity constraint. On the other hand, the returns from solar capacity investments are negative at least until the time $\underline{t}$ at which $p(t)=r p_{K}^{0}+c_{K}+c_{y}>c_{y}$, thus later than $t_{y}$, the time at which $p(t)=c_{y}$. The industry should try to minimize the length of this negative returns period by delaying the beginning of its investments into the solar alternative. The trade-off between these two opposite incentives may result either in a early beginning of the solar investment if the first incentive dominates or conversely in a delayed beginning if the second dominates. This is what happens in type 2 scenarios. In all cases, the solar industry starts to produce even if the energy price is too low to cover the full minimal marginal cost level, that is $r p_{K}^{0}+c_{K}+c_{y}$ and hence faces negative returns over its investments at the early stage of the transition.

Turn now to the scenario 2: $0<t_{K}=t_{y}<t_{X}$. In order that $t_{K}=t_{y}$, it is necessary that:

$$
\lambda_{K}\left(t_{K}\right)=p_{K}^{0} \quad \text { and } c_{x}+\lambda_{X} e^{r t_{K}} \geq c_{y} .
$$

The argument for $\lambda_{K}\left(t_{K}\right)=p_{K}^{0}$ is the same as for the scenario 1 . The inequality is necessary in order that the solar energy production begins once the first piece of equipment is installed.

In scenarios 3 and 4 since $t_{K}=0$, it is now possible that the initial in- 
vestment rate, $k(0)$, be strictly positive and $p_{K}(0)>p_{K}^{0}$ contrary to what happens in scenarios 1 and 2. It is also possible that the inverted $U$ shape pattern of the investment cost dynamics be reduced to a constantly decreasing pattern for $t \geq 0$.

The following Figure 3 illustrates the energy price dynamics and the solar equipment price dynamics in a type 1 scenario.

Figure 3 about here

\section{$5 \quad$ Characterizing the profit maximizing scenario at the equilibrium}

It remains to check the domain of validity of the various scenarios. We are going to describe an algorithmic argument able to address this issue and provide a closed form solution to the present model. The solving procedure will make appear that the characteristics of the solar energy investment plan may be independent from the size of the oil reserves. More precisely, we show that with a sufficiently high initial level of oil reserves, the length of the solar development phase, $T \equiv t_{X}-t_{K}$, and the accumulated capacity at the end of the transition phase, $K_{X}$, do not depend upon $X_{0}$, the initial stock of the non renewable resource.

The idea is quite simple. Consider a scenario of type 1 or 2 starting at $t=0$ with an initial oil endowment $X_{0}$. Let $t_{0}<0$ be an earlier date at which the plan could start with the same phase structure after the date $t=0$. The new plan may start from $t_{0}<0$ with an initial phase of oil consumption between $t_{0}$ and 0 following an energy price path $p(t)=c_{x}+\lambda_{X} e^{r t}, t_{0}<t<0$. This is supported by an initial endowment $X\left(t_{0}\right)$ available at time $t_{0}$, such that:

$$
X\left(t_{0}\right)-X_{0}=\int_{t_{0}}^{0} q^{d}\left(c_{x}+\lambda_{X} e^{r t}\right) d t .
$$

Thus clearly the investment path in solar capacity, the path of oil consumption and the path of solar energy consumption, all after $t=0$, are kept 
unchanged, hence also $T$ and $K_{X}$. We conclude that, once $X_{0}$ is sufficiently large, what happens after $t_{K}$ does not depend upon $X_{0}$ in scenarios 1 and 2 .

The argument does not hold for the scenarios 3 and 4 . Assume that at $t_{0}<0, \lambda_{X}=\lambda_{X}(0)$ being unmodified, the economy is endowed with an oil stock $X\left(t_{0}\right)>X_{0}$ sufficiently large to sustain the oil consumption path of a type 3 or 4 scenario together with the oil consumption path between $t_{0}$ and $t=0$ implied by the energy price $c_{x}+\lambda_{X} e^{r t}$. Note that $t_{y}$ would remain unmodified. Since $k(0)>0$ in such scenarios, $p_{K}(k(0))>p_{K}^{0}$. Thus, when starting from $t_{0}<0$ rather than from $t=0$, the industry should take advantage of this longer delay, start to build its capacity earlier than $t=0$ and reduce the building cost. But such a modification should also change the whole investment path after $t=0$. The consequence would be a different solar production capital at the unchanged time $t=t_{y}$, hence a different solar energy production rate after $t_{y}$. Since $p(t)$ is kept unmodified, the oil consumption rate should be modified too, a contradiction.

The above argument suggests the following dual solving strategy to identify the equilibrium scenario as a function of $X_{0}$ for given other fundamentals of the model, that is to determine first whether the equilibrium scenario is of type 1 or 2 , or a scenario of type 3 or 4 .

To achieve this, assume that $t_{K}=0$, that is take the earliest time at which the solar capacity investment could start, and denote by $T$ the length of the development phase of the solar production capacity before the oil exhaustion. Since $t_{K}=0$, then $t_{X}=T$. Denote by $\lambda_{K}^{0} \geq p_{K}^{0}$ any possible initial value of $\lambda_{K}: \lambda_{K}(0) \equiv \lambda_{K}^{0}$. Let $\lambda_{X}^{0}$ be the shadow value of the oil stock at the beginning of the phase $[0, T)$. For any $\lambda_{X}^{0}$ define $t_{y}$ as:

$$
t_{y}=t_{y}\left(\lambda_{X}^{0}\right)=\left\{\begin{array}{lll}
0 & \text { if } \quad \lambda_{X}^{0} \geq c_{y}-c_{x} \\
\text { solution of: } c_{y}=c_{x}+\lambda_{X}^{0} e^{r t} & \text { if } & \lambda_{X}^{0}<c_{y}-c_{x} .
\end{array}\right.
$$

When $t_{y}>0$ define:

- $\lambda_{K}^{1}\left(t ; \lambda_{K}^{0}\right), 0 \leq t \leq t_{y}$ as the solution of $\dot{\lambda}_{K}=r \lambda_{K}+c_{K}$ with the initial condition $\lambda_{K}(0)=\lambda_{K}^{0}$;

- $\lambda_{K}^{2}\left(t ; \lambda_{K}^{0}\right), t_{y}<t \leq T$ as the solution of $\dot{\lambda}_{K}=r \lambda_{K}+c_{K}+c_{y}$ $\left(c_{x}+\lambda_{X}^{0} e^{r t}\right)$ with the initial condition $\lambda_{K}\left(t_{y}\right)=\lambda_{K}^{1}\left(t_{y} ; \lambda_{K}^{0}\right)$. 
When $t_{y}=0$ define $\lambda_{K}^{2}\left(t ; \lambda_{K}^{0}\right), 0 \leq t \leq T$ as the solution of $\dot{\lambda}_{K}=r \lambda_{K}+c_{K}+$ $c_{y}-\left(c_{x}+\lambda_{X}^{0} e^{r t}\right)$ with the initial condition $\lambda_{K}^{2}\left(0 ; \lambda_{K}^{0}\right)=\lambda_{K}^{0}$.

For any given $\lambda_{K}^{0} \geq p_{K}^{0}$, consider the following problem of finding a triplet $\left(T, \lambda_{X}^{0}, K_{X}\right)$ satisfying the three following conditions:

- Continuity of the energy price at time $T$ :

$$
p^{d}\left(K_{X}\right)=c_{x}+\lambda_{X}^{0} e^{r T} .
$$

- Continuity of $\lambda_{K}(t)$ at $T$, that is $\lambda_{K}(T)$ lying on the saddle branch of the phase diagram in the $\left(K, \lambda_{K}\right)$ plane at $K=K_{X}$ (see Figure 1$)$ :

$$
\lambda_{K}^{X}\left(K_{X}\right)=\lambda_{K}^{2}\left(T ; \lambda_{K}^{0}\right) .
$$

- Continuity of the solar production capital path at time $T$, starting from an initially nil capital, $K(0)=0$ :

$$
K_{X}=\int_{0}^{t_{y}} k^{s}\left(\lambda_{K}^{1}\left(t ; \lambda_{K}^{0}\right)\right) d t+\int_{t_{y}}^{T} k^{s}\left(\lambda_{K}^{2}\left(t ; \lambda_{K}^{0}\right)\right) d t .
$$

We show in Appendix A.3 that the above system has a unique solution, denoted by $\left(T\left(\lambda_{K}^{0}\right), \lambda_{X}^{0}\left(\lambda_{K}^{0}\right), K_{X}\left(\lambda_{K}^{0}\right)\right)$.

Let $K\left(t ; \lambda_{K}^{0}\right)$ be the corresponding solar production capital accumulated at time $t$ :

$$
K\left(t, \lambda_{K}^{0}\right)= \begin{cases}\int_{0}^{t} k^{s}\left(\lambda_{K}^{1}\left(\tau ; \lambda_{K}^{0}\right)\right) d \tau & , 0 \leq t \leq t_{y} \\ K\left(t_{y} ; \lambda_{K}^{0}\right)+\int_{t_{y}}^{t} k^{s}\left(\lambda_{K}^{2}\left(\tau ; \lambda_{K}^{0}\right)\right) d \tau, & t_{y}<t \leq T .\end{cases}
$$

Then $X^{0}\left(\lambda_{K}^{0}\right)$, the corresponding cumulated oil consumption up to time $T$, amounts to:

$$
\begin{aligned}
X^{0}\left(\lambda_{K}^{0}\right)= & \int_{0}^{t_{y}} q^{d}\left(c_{x}+\lambda_{X}^{0}\left(\lambda_{K}^{0}\right) e^{r t}\right) d t \\
& +\int_{t_{y}}^{T}\left\{q^{d}\left(c_{x}+\lambda_{X}^{0}\left(\lambda_{K}^{0}\right) e^{r t}\right)-K\left(t ; \lambda_{K}^{0}\right)\right\} d t .
\end{aligned}
$$


The following Proposition 3 summarizes the properties of the solution of the problem (5.1)-(5.3).

Proposition P. 3 As a function of $\lambda_{K}^{0}$ :

- The solar production capital to be built before the exhaustion of the oil resource is constant: $d K_{X}\left(\lambda_{K}^{0}\right) / d \lambda_{K}^{0}=0$.

- The duration of the solar accumulation capital phase before oil depletion is decreasing: $d T\left(\lambda_{K}^{0}\right) / d \lambda_{K}^{0}<0$.

- The shadow marginal value, $\lambda_{X}^{0}$, of the oil stock necessary to sustain the solution path is increasing and the oil stock is decreasing: $d \lambda_{X}^{0} / d \lambda_{K}^{0}>0$ and $d X^{0}\left(\lambda_{K}^{0}\right) / d \lambda_{K}^{0}<0$.

- Furthermore when $t_{y}>0$, the duration of the accumulation phase without solar energy production is decreasing: $d t_{y}\left(\lambda_{K}^{0}\right) / d \lambda_{K}^{0}<0$.

Proof: See Appendix A.3.

The main point of the Proposition 3 is to show that the solar capital having to be accumulated before oil depletion, $K_{X}$, is independent from the energy transition features. This is a mere consequence of the Hamilton-JacobiBellman equation (HJB therefater) having to apply at $t_{X}$, the exhaustion time of the non renewable resource.

Let $V\left(K_{X}\right)$ be the continuation value of the industry profit maximization program from $t_{X}$ in current value at this time. We have shown in section 3 that $V$ is a function of $K_{X}$ only. Let $\mathcal{H}(t)$ be the current value Hamiltonian. The HJB equation states that at the oil depletion time $t_{X}$ :

$$
\mathcal{H}\left(t_{X}\right) e^{-r t_{X}}=-\frac{\partial}{\partial t_{X}} e^{-r t_{X}} V\left(K_{X}\right)
$$

resulting in:

$$
\begin{array}{r}
p\left(t_{X}\right) q\left(t_{X}\right)-c_{x} x\left(t_{X}\right)-c_{y} y\left(t_{X}\right)-c_{K} K\left(t_{X}\right)-p_{K}\left(t_{X}\right) k\left(t_{X}\right) \\
+\lambda_{K}\left(t_{X}\right) k\left(t_{X}\right)-\lambda_{X}\left(t_{X}\right) x\left(t_{X}\right)=r V\left(K_{X}\right) .
\end{array}
$$


The continuity requirement over the equilibrium energy price trajectory at $t=t_{X}$ implies that: $x\left(t_{X}\right)=0, p\left(t_{X}\right)=p^{d}\left(q\left(t_{X}\right)\right)$ and $q\left(t_{X}\right)=y\left(t_{X}\right)=$ $K\left(t_{X}\right)=K_{X}$ (c.f. Proposition 2). On the other hand, $k\left(t_{X}\right)>0$ implies that $\lambda_{K}\left(t_{X}\right)=p_{K}\left(t_{X}\right)$ (c.f. $\left.(2.3)\right)$. Hence the above HJB equation writes as:

$$
\left[p^{d}\left(K_{X}\right)-\left(c_{K}+c_{y}\right)\right] K_{X}=r V\left(K_{X}\right) .
$$

Since:

$$
V\left(K_{X}\right)=\int_{t_{X}}^{\infty}\left\{\left[p^{d}(K(t))-\left(c_{K}+c_{y}\right)\right] K(t)-p_{K}(t) k(t)\right\} e^{-r\left(t-t_{X}\right)} d t,
$$

time differentiating at $t_{X}$ yields:

$$
\dot{V}\left(K_{X}\right)=-\left[p^{d}\left(K_{X}\right)-\left(c_{K}+c_{y}\right)\right] K_{X}+p_{K}\left(t_{X}\right) k\left(t_{X}\right) .
$$

Thus the HJB equation equivalently states that:

$$
p_{K}\left(t_{X}\right) k\left(t_{X}\right)=r V\left(K_{X}\right)+\dot{V}\left(K_{X}\right) .
$$

The above relation describes the profit maximization condition having to apply in investments in solar production capacity after $t_{X}$ when solar energy is supplying the whole demand.

The HJB equation determines the equilibrium level of $K_{X}$ as a function of the continuation value of the profit maximization plan after the depletion of the oil resource and the current operative profit when solar energy supplies the whole demand. Thus $K_{X}$ appears clearly as determined independently of the features of the transition toward the pure solar energy phase, that is, when $t_{K}=0$, of $T, \lambda_{X}^{0}$ and $\lambda_{K}^{0}$. Note in addition that the condition: $p^{d}\left(K_{X}\right)=c_{x}+\lambda_{X}(T)$ shows that $\lambda_{X}(T)=\lambda_{X}^{0} e^{r T}$ is also independent from $\lambda_{K}^{0}$. Whatever be the detailed characteristics of the capital accumulation plan before oil depletion and the oil resource exploitation plan, the economy must end at the oil depletion time at a price level only determined by $K_{X}$, a capital stock level itself only dependent of the features of the pure solar phase.

We can now identify the critical amount of initial oil endowment above which the equilibrium scenarios are scenarios of types 1 or 2 , and under which they are of types 3 or 4 .

Consider the initial oil endowment $X_{0}=X^{0}\left(p_{K}^{0}\right)$. Starting with this oil endowment, along the equilibrium path, solar production capital investment 
begins immediately and smoothly: $t_{K}=0, \lambda_{K}\left(t_{K}\right)=p_{K}^{0}$ hence $k\left(0^{+}\right)=0$. The transition scenario is a type 1 or a type 2 scenario.

According to the argument developed at the beginning of the present section, for any $X_{0}>X^{0}\left(\lambda_{K}^{0}\right)$, the equilibrium is also a type 1 or a type 2 scenario but now with an initial phase during which only oil is consumed and the investment in solar production capacity is nil: $0<t_{K} \leq t_{y}<t_{X}$, and $k\left(t_{K}^{+}\right)=0$. Depending upon $c_{x}+\lambda_{X}^{0}\left(p_{K}^{0}\right)$ being smaller or larger than $c_{y}$, the equilibrium transition scenario is respectively a scenario 1 or 2 .

Note that when $X_{0} \geq X^{0}\left(p_{K}^{0}\right)$, we get from $\lambda_{X}^{0}\left(p_{K}^{0}\right)$ and $X^{0}\left(p_{K}^{0}\right)$ the calendar time $t_{K}$ at which the energy industry starts to accumulate solar production capital in scenarios of types 1 or 2 . Before $t_{K}=0$, at times $t<0$ measured from $t_{K}=0$, the energy price given by the Hotelling rule is $p(t)=c_{x}+\lambda_{X}^{0}\left(p_{K}^{0}\right) e^{r t}$, and for any given $t<0$, the cumulated oil consumption during the time interval $[t, 0]$ amounts to $\int_{t}^{0} q^{d}\left(c_{x}+\lambda_{X}^{0}\left(p_{K}^{0}\right) e^{r \tau}\right) d \tau$. Thus the date $t_{K}$ now measured in calendar time is the length of the phase during which the cumulated oil consumption amounts to $X_{0}-X^{0}\left(p_{K}^{0}\right)$, that is $-t_{K}$, in times measured from $t_{K}=0$, solves:

$$
\int_{-t_{K}}^{0} q^{d}\left(c_{x}+\lambda_{X}^{0}\left(p_{K}^{0}\right) e^{r t}\right) d t=X_{0}-X^{0}\left(p_{K}^{0}\right)
$$

An immediate implication of the above result is that for initial endowments $X_{0}<X^{0}\left(p_{K}^{0}\right)$, then $t_{K}=0$ and $\lambda_{K}^{0}=p_{K}(0)>p_{K}^{0}$. The corresponding scenario is of type 3 or 4 . The lower is $X_{0}$, the higher is $\lambda_{K}^{0}$, hence $p_{K}(0)$ and $k(0)$, and the higher is $\lambda_{X}^{0}$, here equal to $\lambda_{X}$. Again depending upon $c_{x}+\lambda_{X}^{0}\left(\lambda_{K}^{0}\right)$ being smaller or larger than $c_{y}$, the equilibrium transition scenario is respectively a scenario 3 or a scenario 4 .

The following proposition summarizes these findings.

Proposition P. 4 Let $\left(T\left(\lambda_{K}^{0}\right), K_{X}\left(\lambda_{K}^{0}\right), \lambda_{X}^{0}\left(\lambda_{K}^{0}\right)\right)$ be the unique solution of the system (5.1)-(5.3) and let $X^{0}\left(\lambda_{K}^{0}\right)$ be the corresponding cumulated oil consumption. Then:

1. If $X_{0}>X^{0}\left(p_{K}^{0}\right)$, the equilibrium path begins with a first phase $\left[0, t_{K}\right)$ of 
only oil production without investment into the solar energy alternative and:

- If $\lambda_{X}^{0}\left(p_{K}^{0}\right)<c_{y}-c_{x}$, then $t_{K}<t_{y}$ and the energy transition follows the Scenario 1, with a second phase $\left[t_{K}, t_{y}\right)$ of investment into solar capacity without solar energy production, only oil being consumed, followed by a phase $\left[t_{y}, t_{X}\right)$ of investment and joint use of both energy sources.

- If $\lambda_{X}^{0}\left(p_{K}^{0}\right)>c_{y}-c_{x}$, then $t_{K}=t_{y}$ and the energy transition follows the Scenario 2, with a single phase of investment during which both energy sources are exploited until oil depletion.

2. If $X_{0}<X^{0}\left(p_{K}^{0}\right)$, then there exists some $\lambda_{K}^{0}>p_{K}^{0}$ such that $X^{0}\left(\lambda_{K}^{0}\right)=$ $X_{0}$ and the development of the solar energy alternative starts immediately from $t=0$ meaning that $t_{K}$ is reduced to zero and:

- If $\lambda_{X}^{0}\left(\lambda_{K}^{0}\right)<c_{y}-c_{x}$, then $t_{y}>0$ and the energy transition follows the Scenario 3, composed of a first phase $\left[0, t_{y}\right)$ of investment into solar capacity without solar energy production, followed by a phase $\left[t_{y}, t_{X}\right)$ of investment and joint use of both energy sources.

- If $\lambda_{X}^{0}\left(\lambda_{K}^{0}\right)>c_{y}-c_{x}$, then $t_{K}=t_{y}=0$ and the energy transition corresponds to the Scenario 4, that is a single phase of investment and joint production from both energy sources until oil depletion.

\section{Discussion and policy implications}

We first show that the main properties of the equilibrium paths still hold under alternative standard assumptions. Next we examine the impacts of different policies over the equilibrium paths.

\subsection{Alternative standard assumptions}

The main characteristics of the transition scenarios result from the impossibility to build instantaneously a solar production capital at a constant average cost. Would this be possible there would not exist a transition phase 
during which both resources are simultaneously exploited. The oil would be first exploited up to the time at which it is exhausted and at this date the solar capital would be built once and for all. Thus the energy price would be first increasing during the oil extraction phase and next constant during the solar phase. Since the average cost of the solar capital is constant, discounting implies to delay the investment as far as possible, that is up to its once and for all installation. However such a model implicitly assumes that the instantaneous rate of the solar equipment can be infinite, an audacious assumption. ${ }^{6}$

The sensible counterpart of a constant average cost assumption in the solar equipment industry is the correlative assumption of a bounded production capacity. Let $c_{s}$ be the assumed constant average cost of the equipment industry and $\bar{k}$ its production capacity assumed to be constant through time to simplify, hence a total cost at time $t$ equal to $c_{s} k(t), 0 \leq k(t) \leq \bar{k}$.

In this modified model, the price $p_{K}(t)$ at which the energy industry purchases its solar equipment is higher than $c_{s}$ and when positive, $k(t)=\bar{k}$, that is the investment rate in solar energy production capacity is constant during the whole period of capacity building. ${ }^{7}$ All along the accumulation process, the energy industry must cover the full marginal cost $r p_{K}(t)+c_{K}+c_{y}$, thus $K\left(t_{X}\right)=K_{X} \leq \hat{K}_{s}$, the long run capacity being here given by $p^{d}\left(\hat{K}_{s}\right)=$ $r c_{s}+c_{K}+c_{y}$. While in the convex cost model, this inequality holds at as a strict inequality, it may hold as an equality in the constant average cost model. The HJB equation must thus be modified as follows. Let $\omega$ be the Lagrange multiplier associated to the constraint $\hat{K}_{s}-K_{X} \geq 0$. By complementarity slackness, we get $\omega \geq 0$ and $\omega\left(\hat{K}_{s}-K_{X}\right)=0$. The relevant HJB equation for the constant average cost model reads:

$$
\left[p^{d}\left(K_{X}\right)-\left(c_{K}+c_{y}\right)\right] K_{X}=r V\left(K_{X}\right)-\omega .
$$

If the constraint does not bind, $\omega=0$ and (5.6) determines $K_{X}$. All the other main characteristics of the equilibrium paths are left unchanged excepted that now appear two pure solar phases. During the first one of finite duration the solar energy production capital is brought from $K_{X}$ up to its long run equilibrium $\hat{K}_{s}$. Thus denoting by $t_{k}$ the time at which ends the investment

\footnotetext{
${ }^{6}$ In discrete time models, the impossibility of infinite instantaneous production rates tends to be diluted.

${ }^{7}$ We show in Appendix A.4 that we cannot have $p(t)=c_{s}$ over any non degenerate time interval during which the investment rate is positive.
} 
in solar equipment, then $t_{k}-t_{X}=\left(\hat{K}_{s}-K_{X}\right) / \bar{k}$. The second phase of infinite duration, $\left(t_{k}, \infty\right)$, is the long run stationary solar phase: $K(t)=\hat{K}_{s}$ and $y(t)=\hat{K}_{s}, t \geq t_{k}{ }^{8}$.

If the constraint binds, $K_{X}=\hat{K}_{s}$ and $\left[p^{d}\left(\hat{K}_{s}\right)-\left(c_{K}+c_{y}\right)\right] \leq r V\left(\hat{K}_{s}\right)$. In this last case, the first pure solar phase is reduced to zero, $\bar{k}$ being sufficiently high for the industry to be able to accumulate the long run capacity $\hat{K}_{s}$ at the oil depletion time. Note that, like in the initial model, the HJB equation and the continuity of the energy price path imply that the solar capacity accumulated at the exhaustion time of the fossil fuel energy depends only upon the characteristics of the supply of capital equipment, here $c_{s}$ and $\bar{k}$, together with the maintenance cost, $c_{K}$, and the operative cost, $c_{y}$.

The other seemingly strong assumption of our model is the presumed constant average cost of non renewable energy provision. It implies in particular that the whole stock of oil is exploited. A more realistic assumption would be to consider average extraction cost functions increasing with past cumulated consumption. Assume that $c_{x} \equiv c_{x}(X)$, defined over $\left(0, X_{0}\right]$, is twice continuously differentiable, decreasing and convex, with $c_{x}\left(0^{+}\right)=+\infty$ to insure that the whole stock $X_{0}$ will not be mined. The new problem is to determine that part of $X_{0}$ which will be consumed.

To determine the grade $X\left(t_{X}\right)$ at which ends the oil extraction, we may use the HJB equation which reads now:

$$
\begin{aligned}
p\left(t_{X}\right) q\left(t_{X}\right)- & c_{x}\left(X\left(t_{X}\right)\right) x\left(t_{X}\right)-c_{K} K\left(t_{X}\right)-p_{K}\left(t_{X}\right) k\left(t_{X}\right) \\
& +\lambda_{K}\left(t_{X}\right) k\left(t_{X}\right)-\lambda_{X}\left(t_{X}\right) x\left(t_{X}\right)=r V\left(K_{X}\right) .
\end{aligned}
$$

Again the continuity of the equilibrium energy price path at $t=t_{X}$ implies that $x\left(t_{X}\right)=0, p\left(t_{X}\right)=p^{d}\left(q\left(t_{X}\right)\right)$ and $q\left(t_{X}\right)=y\left(t_{X}\right)=K\left(t_{X}\right)$. Again too, $k\left(t_{X}\right)>0$ implies that $\lambda_{K}\left(t_{X}\right)=p_{K}\left(t_{X}\right)$. We conclude that (5.6) is left unmodified, hence that $K\left(t_{X}\right)$ depend only upon the characteristics of the supply function $k^{s}$ and the maintenance and operative costs of solar capital as in the initial model.

Furthermore the mining rent must be nil for the last exploited grade. Hence from the energy price continuity, $p\left(t_{X}\right)=p^{d}\left(K_{X}\right)$, and the zero rent

\footnotetext{
${ }^{8}$ Formally the other evident difference is that now in the scenarios 1 and 2 when begins the solar capital accumulation process, $p_{K}\left(t_{K}\right)=c_{s}$ and $k\left(t_{K}^{+}\right)=\bar{k}$ and not $k\left(t_{K}^{+}\right)=0$.
} 
condition, the stock of oil left underground, $X\left(t_{X}\right)$, appears as the solution of $c_{x}(X)=p^{d}\left(K\left(t_{X}\right)\right)$. The other qualitative properties of the scenarios are the same. For example for a sufficiently cheap least costly grade $c_{x}\left(X_{0}\right)$, the equilibrium scenarios are of type 1 or 2 , while for higher least costs levels, they are of type 3 or 4 .

Here again the details of the investment phases preceding the exhaustion of the non renewable resource between $t_{K}$ and $t_{X}$ in equilibrium scenarios of type 1 or 2 are independent from the oil endowment to some extent. Assume that for $X_{0}$ and a given cost function $c_{x}(X)$, the investment in solar equipment starts at some time $t_{K}>0$. Then consider any alternative oil endowment $X_{0}^{\prime}, X\left(t_{K}\right)<X_{0}^{\prime}<X_{0}$, and let $t_{0}^{\prime}$ be the time at which $X(t)=X_{0}^{\prime}$ along the equilibrium path, $0<t_{0}^{\prime}<t_{K}$. Then for this new initial endowment and the same non renewable energy cost function, that is the restriction of $c_{x}(X)$ to $\left(0, X_{0}^{\prime}\right]$, the new equilibrium path is the initial path from $t_{0}^{\prime}$ onward: the value of any variable $z$ at time $t>t_{0}^{\prime}$ is the value of the same variable at time $t-t_{0}^{\prime}$ along the new equilibrium path starting from $X_{0}^{\prime}$ at time $t=0$.

\subsection{Policy implications}

The peak price of energy is attained at the depletion time of the oil resource. Note that being given by $p\left(t_{X}\right)=p^{d}\left(K_{X}\right)$ at the equilibrium, the peak price is independent from the features of the transition toward pure renewable energy. After oil exhaustion, the energy price either continuously decreases or decreases during the finite duration phase of completion of the long run solar energy production capital when the equipment industry supplies capital at a constant average cost with an upper limit over its production rate.

The robust results of Proposition 4 show that the features of the solar development phases may be largely independent from the availability of the non renewable resource for sufficiently high initial endowments. Investment into the solar substitute is delayed by a higher oil reserves but once started, the investment path in solar equipment together with oil consumption path are the same, hence shifting later in time the same level of the peak price.

The above results have interesting implications in the discussion of the so- 
called Green Paradox (Sinn, 2008 and 2012). ${ }^{9}$ More precisely, they suggest that fossil taxation policies should have quite different impacts upon the equilibrium paths than carbon free energy promoting policies.

The most common taxation system is an unitary tax on the fossil fuel consumption. It is well known that such a tax growing at a proportional rate equal to the interest rate has no effect on the equilibrium path. Let $\tau$ be the initial unitary tax and $\tau(t)=\tau e^{r t}$ its amount at time $t$. Denote by $\lambda_{X}(\tau)$ the initial mining rent under this taxation regime. Clearly the oil consumption path and the solar equipment path are left unmodified provided that $\tau+\lambda_{X}(\tau)=\lambda_{X}$ where $\lambda_{X} \equiv \lambda_{X}(0)$ is the initial mining rent in the tax free equilibrium. The only effect of such a tax is to reduce the mining rent by the same amount, a pure redistributive effect upon the mines owners.

An unitary tax growing at a constant proportional rate $\rho$ higher than the interest rate should increase the early fuel consumption rates and decrease the late ones since the exploited oil stock is constant in the present model. However in stock dependent cost models, the cumulated oil consumption decreases because the extraction end at a less costly grade. The HJB equation with a tax growing at a rate $\rho>r$ reads:

$$
\begin{array}{r}
p\left(t_{X}\right) q\left(t_{X}\right)-c_{x} x\left(t_{X}\right)-\tau e^{\rho t_{X}} x\left(t_{X}\right)-\lambda_{X}\left(t_{X}\right) x\left(t_{X}\right)-c_{K} K\left(t_{X}\right) \\
-p_{K}\left(t_{X}\right) k\left(t_{X}\right)+\lambda_{K}\left(t_{X}\right) k\left(t_{X}\right)=r V\left(K\left(t_{X}\right)\right) .
\end{array}
$$

The argument used in section 5 shows that (5.6) still holds. Note that the same argument also holds when $c_{x}\left(X\left(t_{X}\right)\right) x\left(t_{X}\right)$ is substituted to $c_{x} x\left(t_{X}\right)$ in the above equation. However in the stock dependent cost case the zero mining rent condition for $X\left(t_{X}\right)$ under taxation implies that the last exploited grade must satisfy $p^{d}\left(K_{X}\right)-\tau e^{\rho t_{X}}=c_{x}\left(X\left(t_{X}\right)\right)$. Since $p^{d}\left(K_{X}\right)$ does not depend upon the tax rate, then $X\left(t_{X}\right)$ should be higher under the tax regime than under the tax free regime.

Solar energy promotion can take several forms: subsidies for the purchase of equipment, reduced interest rates, subsidies to maintenance and operative costs or higher selling prices of solar energy than fossil energy prices. Another common promotion device is to impose constraints on the energy mix supply, for example a fixed or a proportional mandate over the use of solar

\footnotetext{
${ }^{9}$ For recent surveys of partial equilibrium analysis results see Hoel (2012) and Van der Ploeg (2013).
} 
energy, either immediately of at some given future date. We have focused on promoting policies reducing either the maintenance cost, $c_{K}$, or the operative cost, $c_{y}$. Such reductions may result from direct subsidies to solar energy generation, equipment purchase subsidies or subsidized solar energy tariffs.

It can be shown ${ }^{10}$ that in a scenario of type 1 or 2 , a decrease of $c_{K}$ or $c_{y}$ induces an increase of $K_{X}$, the accumulated solar production capital at the beginning of the pure solar phase, a decrease of $\lambda_{X}^{0}$ and has ambiguous effects over $T$, the length of the transition phase toward pure renewable energy. A decrease of $\lambda_{X}^{0}$ means a decrease of $t_{K}$ and $t_{y}$. These results should probably be expected. Promoting renewable energy policies favor an earlier start of the investment process and an earlier introduction of solar energy in the energy mix together with a higher accumulation of solar production capital. This last result stands in contrast with oil taxation, a fossil fuel tax having no impact upon the accumulated solar production capacity at the end of oil exploitation.

\section{Conclusion}

We have shown that under adjustment costs, the transition from non renewable to renewable energy is smooth. The energy price is singled peaked, the peak occurring at the time of exhaustion of the non renewable resource. Although the Hotelling rule drives the energy price dynamics before and during the transition, the peak is independent of the initial nonrenewable resource endowment. Equivalently, the capital required to use the renewable resource at the depletion time of the non renewable resource is independent of the initial resource stock. Only the characteristics of the energy demand function, the equipment supply function, the interest rate and the maintenance and operative cost structure matter to determine the peak price and/or the accumulated capital at the exhaustion time. This conclusion is robust to alternative assumptions about the shape of the supply function of equipment or about the extraction costs of the non renewable resource.

\footnotetext{
${ }^{10}$ The details of the calculations are available upon request from the authors.
} 
An immediate policy implication of the above results is that under a fossil fuel taxation regime, the peak price of energy is left unmodified, although the price path is modified before the fossil fuel exhaustion together with the depletion time. The renewable energy generation capital accumulated at the exhaustion time is independent of the tax rate, although again the investment path is modified during the energy transition. On the contrary, subsidies to renewable energy modify both the peak price of energy and the capital accumulated at the exhaustion time together with the long run level of renewable energy production capacity.

One strong motivation for developing renewable energy alternatives is climate change mitigation. An explicit account of the polluting nature of fossil fuels inside our model should impact in more or less complex ways both the beginning time and the speed of development of the green energy alternatives. In a carbon pollution accumulation framework not considering the scarcity of fossil fuels but close to the present model in the specification of the renewable production capacity building process, Fischer et al. (2004) conclude that the long run accumulated renewable capacity is independent from the details of the transition toward clean energy, a finding in line with our results. With a fixed renewable production capacity, Gronwald et al. (2013) conclude that moderate subsidies should enhance the transition toward clean energy, in accordance with our results. Most policy proposals to mitigate climate change are designed in order to accelerate the transition toward clean energies, but may have unintended negative side effects, an important issue deserving more theoretical and empirical research. 


\section{References}

ACEMOGLU D., AGHION P., BURSZTYN L. and D. HEMOUS, (2012), The Environment and Directed Technical Change, American Economic Review, 102(1), 131-66.

AMIGUES J. P., FAVARD P., GAUDET G. and M. MOREAUX, (1998), On the Optimal Order of Natural Resource Use when the Capacity of the Inexhaustible Substitute is Limited, Journal of Economic Theory, 80(1), 153170 .

CAIRNS R. D. and P. LASSERRE, (1991), The Role of Investment in Multiple-Deposit Extraction: Some Results and Remaining Puzzles, Journal of Environmental Economics and Management, 21(1), 52-66.

CAMPBELL H. F., (1980), The Effect of Capital Intensity on the Optimal Rate of Extraction of a Mineral Deposit, The Canadian Journal of Economics, 13(2), 349-356.

FISCHER C., TOMAN M. and C. WITHAGEN, (2004), Optimal Investment in Clean Production Capacity, Environmental and Resource Economics, 28(3), 325-345.

FOUQUET R., (2008), Heat, Power and Light: Revolutions in Energy Services. Edward Elgar Publications. Cheltenham, UK, and Northampton, MA, USA.

GAUDET G., (1983), Optimal Investment and Adjustment Costs in the Economic Theory of the Mine, The Canadian Journal of Economics, 16(1), $39-51$.

GAUDET G., (2007), Natural Resource Economics under the Rule of Hotelling, The Canadian Journal of Economics, 40(4), 1033-1059.

GOULD J. P., (1968) Adjustment Costs in the Theory of Investment of 
the Firm, Review of Economic Studies, 35, 47-55.

GRONWALD M., N. V. LONG and L. ROEPKE, (2013), Simultaneous Supplies of Dirty and Green Fuels with Capacity Constraints: Is There a Green Paradox?, CESIfo WP 4360.

HERFINDAHL O. C. and A. V. KNEESE, (1974), Economic Theory of Natural Resources, Merril, Columbus.

HOEL M., (2012), Carbon Taxes and the Green Paradox, in R. W. Hahn and A. Ulph, eds, Climate Change and Common Sense: Essays in Honor of Tom Schelling, Oxford University Press, Oxford.

HOLLAND S. (2003), Extraction Capacity and the Optimal Order of Extraction, Journal of Environmental Economics and Management, 64(3), 364-376.

HOTELLING H., (1931), The Economics of Exhaustible Resources, The Journal of Political Economy, 39(2), 137-175.

IEA (2013), Key world energy statistics, Paris, www.iea.org.

KEMP M. C. and N. V. LONG, (1980), On Two Folk Theorems Concerning the Extraction of Exhaustible Resources, Econometrica, 48(3), 663-673.

LASSERRE P., (1986), Exhaustible Resource Extraction with Capital, in A.D. Scott, ed., Progress in National Resource Economics, Oxford University Press, Oxford.

LIVERNOIS J., (2009), On the Empirical Significance of the Hotelling Rule, Review of Environmental Economics and Policy, 3(1), 22-41.

LUCAS R., $\left(1967^{a}\right)$, Optimal Investment Policy and the Flexible Accelerator, International Economic review, 8(1), 78-85.

LUCAS R., $\left(1967^{b}\right)$, Adjustment Costs and the Theory of Supply, Journal 
of Political Economy, 75(1), 321-334.

NAKICENOVIC N., A. GRUBLER and A. McDONALD, (1998), Global Energy Perspectives, Cambridge University Press, Cambridge.

OLSEN T. E., (1989), Capital Investments and Resource Extraction from Non-Identical Deposits, Journal of Environmental Economics and Management, 17(1), 127-239.

PLOEG, F van der, (2013), Cumulative Carbon Emissions and the Green Paradox, Annual Review of Resource Economics, 5(1), 281-300.

POWELL S. G. and S. S. OREN, (1989), The Transition to Non depletable Energy: Social Planning and Market Models of Capacity Expansion Operations Research, 37(3), 373-383.

PUU T., (1977), On the Profitability of Exhausting Natural Resources, Journal of Environmental Economics and Management, 4, 185-199.

SINN H. W., (2008), Public Policies against Global Warming: A Supply Side Approach, International Tax and Public Finance, 15, 360-394.

SINN H. W., (2012), The green paradox: A supply-side approach to global warming, Cambridge University Press, Cambridge.

SMULDERS S. H., TSUR Y. and A. ZEMEL, (2012), Announcing Climate Policy: Can a Green Paradox Arise without Scarcity, Journal of Environmental Economics and Management, 64(3), 364-376.

SWITZER S. and S. SALANT. (1986). Expansion Optimale de Capacité par des Exploitants Prévoyants d'une Ressource Non Renouvelable. in G. Gaudet and P. Lasserre (eds.) Ressources Naturelles et Théorie Economique, 188-210, Les Presses de L'Université Laval, Quebec.

TREADWAY A. B., (1969), On Rational Entrepreneurial Behavior and the Demand for Investment, Review of Economic Studies, 36, 227-39. 
TSUR Y. and A. ZEMEL, (2011), On the Dynamics of Competing Energy Sources, Automatica, 47(1), 1357-1365.

WIRL F., (1991), (Monopolistic) Resource Extraction and Limit Pricing: The Market Penetration of Competitively Produced Synfuels, Environmental and Resource Economics, 1, 157-178.

WRIGLEY E.A., (2010), Energy and the English Industrial revolution, Cambridge University Press, Cambridge. 


\section{Appendix}

\section{A.1 Appendix A.1}

Since $k(t)>0$ by assumption, $\dot{K}(t)>0$. On the other hand, $\dot{\lambda}_{K}>/=$ $/<0$ depending upon $\lambda_{K}>/=/<v(K(t))$ where $v(K) \equiv\left[p^{d}(K)-c_{y}-\right.$ $\left.c_{K}\right] / r=\beta_{K}(t) / r$. As shown before, $v(K(t))$ is the total net marginal surplus in current value from $t$ onwards if the solar production capacity and hence the solar energy production level would be kept constant after $t$. Thus $v(K)$ measures the capacity rent resulting from a constant capacity level $K$. Since $d p^{d}(q) / d q<0$ and $\lim _{q \downarrow 0} p^{d}(q)=+\infty$ under our demand assumption, we get immediately $\lim _{K \downarrow 0} v(K)=+\infty$ and $d v(K) / d K<0$. Last, $\bar{K}$ appears equivalently as the solution of $v(K)=0$ while $v(K)<0$ for $K>\bar{K}$.

It is easily checked that the saddle branch converging toward $\left(\hat{K}, p_{K}^{0}\right)$ is the only equilibrium solar capacity path starting from $K_{X}<\hat{K}$. There exist two other main types of trajectories solution of (3.1)-(3.2). A first kind of trajectories initiates under the locus $\dot{\lambda}_{K}=0$ and then move in finite time above this curve inside a region where $\dot{\lambda}_{K}>0$ and thus $\dot{k}>0$ since $k=$ $k^{s}\left(\lambda_{K}\right)$ and $d k^{s}\left(p_{K}\right) / d p_{K}>0$. Furthermore in this region: $p_{K}(k)=\lambda_{K}>$ $v(K)$. Since $y(t)=K(t)$ increases permanently, $p(t)$ decreases implying that:

$$
\begin{aligned}
\int_{t}^{\infty} e^{-r(\tau-t)}\left[p(\tau)-c_{y}-c_{K}\right] d \tau & <\int_{t}^{\infty} e^{-r(\tau-t)}\left[p(t)-c_{y}-c_{K}\right] d \tau \\
& =\frac{p^{d}(K(t))-c_{y}-c_{K}}{r}=v(K(t)) .
\end{aligned}
$$

Thus above the curve $v(K)$ :

$$
p_{K}(k(t))>v(K(t))>\int_{t}^{\infty} e^{-r(\tau-t)}\left[p(\tau)-c_{y}-c_{K}\right] d \tau .
$$

The marginal cost of an investment into an increase of the capacity would be higher than the total marginal gain from such an investment which cannot be profit maximizing.

The other kind of trajectories starts from below the saddle branch and then moves towards the horizontal $p_{K}^{0}$ in finite time. Consider such a trajectory ending at some capacity level $K_{a}$ as illustrated upon Figure 1 . Let $t_{a}$ be 
that time when $K\left(t_{a}\right)=K_{a}$. Since along such trajectories $\lambda_{K}(t)<v(K(t))$ we get at $t_{a}$ :

$$
\lambda_{K}\left(t_{a}\right)=p_{K}^{0}<v\left(K_{a}\right)=\int_{t}^{\infty} e^{-r\left(\tau-t_{a}\right)}\left[p^{d}\left(K_{a}\right)-c_{y}-c_{K}\right] d \tau .
$$

A slight investment effort $d k>0$ above 0 would generate a surplus gain higher than its cost, showing that such a choice of an investment policy into solar production capacity building could not be efficient for the energy industry.

\section{A.2 Appendix A.2}

Consider the transition scenario 1 where $0<t_{K}<t_{y}<t_{X}$. During the phase $\left[t_{K}, t_{y}\right), \dot{\lambda}_{K}=r \lambda_{K}+c_{K}>0$. Integrating this equation over $\left[t_{K}, t\right), t \leq t_{y}$ yields:

$$
\lambda_{K}(t)=e^{r\left(t-t_{K}\right)} p_{K}^{0}+\frac{c_{K}}{r}\left(e^{r\left(t-t_{K}\right)}-1\right) \quad t \in\left[t_{K}, t_{y}\right) .
$$

Next, (2.6) together with the expression of $\gamma_{K}(t)$ resulting from (2.2), yields for $t \geq t_{y}$ :

$$
\dot{\lambda}_{K}(t)=r \lambda_{K}(t)+c_{y}+c_{K}-c_{x}-\lambda_{X} e^{r t} .
$$

Note that since $p\left(t_{y}\right)=c_{x}+\lambda_{X} e^{r t}=c_{y}, \dot{\lambda}_{K}\left(t_{y}^{-}\right)=\dot{\lambda}_{K}\left(t_{y}^{+}\right), \lambda_{K}(t)$ is both time continuous and differentiable at $t_{y}$. Integrating over a time interval $\left[t_{0}, t\right)$, $t_{y} \leq t_{0}<t$ gets:

$$
\lambda_{K}(t)=\lambda_{K}\left(t_{0}\right) e^{r\left(t-t_{0}\right)}+\frac{c_{y}+c_{K}-c_{x}}{r}\left(e^{r\left(t-t_{0}\right)}-1\right)-\lambda_{X} e^{r t}\left(t-t_{0}\right) .
$$

Thus:

$$
\dot{\lambda}_{K}(t)=\pi\left(t_{0}\right) e^{r\left(t-t_{0}\right)}-r \lambda_{X} e^{r t}\left(t-t_{0}\right),
$$

where $\pi\left(t_{0}\right) \equiv r \lambda_{K}\left(t_{0}\right)+c_{y}+c_{K}-c_{x}-\lambda_{X} e^{r t_{0}}$. If $\pi\left(t_{0}\right)<0$ then $\dot{\lambda}_{K}(t)<0$. In the reverse case, there exists $\bar{t}$ solution of $\dot{\lambda}_{K}(t)=0$ that is of:

$$
t=t_{0}+\frac{\pi\left(t_{0}\right)}{r \lambda_{X} e^{r t_{0}}} .
$$


Investment into solar energy should take place before that time $\underline{t}$ at which $p(t)=r p_{K}^{0}+c_{y}+c_{K}$, that is $t_{K}<\underline{t} . \underline{t} \geq 0$ requires that $\lambda_{X}$ be sufficiently low for: $c_{x}+\lambda_{X} e^{r \underline{t}}=r p_{K}^{0}+c_{K}+c_{y}$. To show the claim $t_{K}<\underline{t}$, assume to the contrary that $\underline{t} \leq t_{K}$, thus $p\left(t_{K}\right) \geq c_{y}+c_{K}+r p_{K}^{0}$. Then, either the investment process starts smoothly at $t_{K}$, that is $k\left(t_{K}^{+}\right)=0$, either its starts abruptly so that $k\left(t_{K}^{+}\right)>0$. In the first case, $\lambda_{K}\left(t_{K}^{+}\right)=p_{K}^{0}$ implies that $\dot{\lambda}_{K}\left(t_{K}^{+}\right)=r \lambda_{K}\left(t_{K}^{+}\right)+c_{K}+c_{y}-p\left(t_{K}\right)<0$. Thus there exists a time interval $\left(t_{K}, t_{K}+\delta\right), \delta>0$ such that $\lambda_{K}(t)<p_{K}^{0}$ while $k(t)>0$, hence a contradiction. In the second case, $p_{K}\left(t_{K}^{+}\right)>p_{K}^{0}$. Since $\lambda_{K}(t)$ is time continuous, there exists a time interval $\left(t_{K}-\delta, t_{K}\right), \delta>0$, such that $\lambda_{K}(t)>p_{K}^{0}$. Thus the industry should have invested during this time interval, also a contradiction.

It is easily verified that necessarily, $t_{y}<\underline{t}<\bar{t}$. Since $\beta_{K}(t)=p(t)-$ $\left(c_{y}+c_{K}\right), \underline{t}$ is equivalently defined as the solution of $p_{K}^{0}=\beta_{K}(t) / r$. Note that $\gamma_{K}(t)=0$, for $t \in\left[t_{K}, t_{y}\right)$ implies that $\beta_{K}(t)=-c_{K}$ during the first investment phase. For $t \in\left[t_{y}, t_{X}\right), \dot{p}(t)>0 \Longrightarrow \dot{\beta}_{K}(t)>0$. Thus, $\beta_{K}\left(t_{y}\right)<$ $0<\beta_{K}(\underline{t})=r p_{K}^{0}$ implies that $t_{y}<\underline{t}$. On the other hand, $\dot{\lambda}_{K}(t)>0$ within the time interval $\left[t_{K}, \bar{t}\right)$ implies that $\lambda_{K}(\bar{t})=\beta_{K}(\bar{t}) / r>p_{K}^{0}=\beta_{K}(\underline{t}) / r$ and thus $\underline{t}<\bar{t}$.

Next, it is easily checked that the time derivative of $\lambda_{K}(t)$ at $t_{y}$, although continuous at $t_{y}$, has a kink at this time. At $t_{y}^{-}$:

$$
\ddot{\lambda}_{K}\left(t_{y}^{-}\right)=r^{2} \lambda_{K}\left(t_{y}^{-}\right) .
$$

On the other hand, at $t_{y}^{+}$:

$$
\ddot{\lambda}_{K}\left(t_{y}^{+}\right)=r^{2} \lambda_{K}\left(t_{y}^{+}\right)-r \lambda_{X} e^{r t_{y}^{+}} .
$$

Since $\lambda_{K}(t)$ is continuous at $t_{y}$, then: $\ddot{\lambda}_{K}\left(t_{y}^{-}\right)>\ddot{\lambda}_{K}\left(t_{y}^{+}\right)$.

Last, to check that $\bar{t}<t_{X}$, evaluate (2.6) at the right end limit $t=t_{X}^{+}$:

$$
\dot{\lambda}_{K}\left(t_{X}^{+}\right)=r \lambda_{K}\left(t_{X}\right)+c_{K}+c_{y}-p^{d}\left(K_{X}\right) .
$$

Performing the same evaluation at the left end limit $t=t_{X}^{-}$:

$$
\dot{\lambda}_{K}\left(t_{X}^{-}\right)=r \lambda_{K}\left(t_{X}\right)+c_{K}+c_{y}-\left(c_{x}+\lambda_{X} e^{r t}\right) .
$$

The price path having to be time continuous at $t_{X}: c_{x}+\lambda_{X} e^{r t_{X}}=p^{d}\left(K_{X}\right)$, hence $\dot{\lambda}_{K}\left(t_{X}^{-}\right)=\dot{\lambda}_{K}\left(t_{X}^{+}\right) . \quad \lambda_{K}(t)$ is not only continuous but also time differentiable at $t=t_{X}$. Since $K_{X}<\hat{K}, \dot{\lambda}_{K}\left(t_{X}^{-}\right)=\dot{\lambda}_{K}\left(t_{X}^{+}\right)<0$. This shows the 
claim of the Corollary 1. Since $\dot{\lambda}_{K}(t)<0$ only if either $t>\bar{t}$ or $t \geq 0$ the Corollary 1 implies that $\bar{t}<t_{X}$ when $\bar{t}$ is defined.

\section{A.3 Appendix A.3}

\section{A.3.1 Partial derivatives of $\lambda_{K}(t)$}

During the time interval $\left[t_{K}, t_{y}\right), \lambda_{K}^{1}(t)$ is defined by (A.2.1). Denote by $\pi_{0} \equiv r \lambda_{K}^{0}+c_{K}$, then:

$$
\begin{aligned}
& \frac{\partial \lambda_{K}^{1}(t)}{\partial t}=-\frac{\partial \lambda_{K}^{1}(t)}{\partial t_{K}}=\pi_{0} e^{r\left(t-t_{K}\right)}>0 \\
& \frac{\partial \lambda_{K}^{1}(t)}{\partial \lambda_{K}^{0}}=e^{r\left(t-t_{K}\right)} .
\end{aligned}
$$

During the joint exploitation phase of oil and solar energy $\left[t_{y}, t_{X}\right), \lambda_{K}^{2}(t)$ is given by:

$$
\lambda_{K}^{2}(t)=e^{r\left(t-t_{y}\right)} \lambda_{K}^{2}\left(t_{y}\right)+\frac{c_{y}+c_{K}-c_{x}}{r}\left(e^{r\left(t-t_{y}\right)}-1\right)-\lambda_{X} e^{r t}\left(t-t_{y}\right) .
$$

Since $\lambda_{K}(t)$ is a continuous time function at $t=t_{y}$ :

$$
\lambda_{K}^{2}\left(t_{y}\right)=\lambda_{K}^{1}\left(t_{y}\right)=e^{r\left(t_{y}-t_{K}\right)} \lambda_{K}^{0}+\frac{c_{K}}{r}\left(e^{r\left(t_{y}-t_{K}\right)}-1\right)
$$

thus:

$$
\begin{aligned}
\lambda_{K}^{2}(t)= & \lambda_{K}^{0} e^{r\left(t-t_{K}\right)}+\frac{c_{K}}{r}\left(e^{r\left(t-t_{K}\right)}-1\right)+\frac{c_{y}-c_{x}}{r}\left(e^{r\left(t-t_{y}\right)}-1\right) \\
& -\lambda_{X} e^{r t}\left(t-t_{y}\right) .
\end{aligned}
$$

(A.3.3) defines $\lambda_{K}^{2}(t)$ during the time interval $\left(t_{y}, t_{X}\right)$ and:

$$
\begin{aligned}
\frac{\partial \lambda_{K}^{2}(t)}{\partial t} & =\left(r \lambda_{K}^{0}+c_{K}\right) e^{r\left(t-t_{K}\right)}+\left(c_{y}-c_{x}\right) e^{r\left(t-t_{y}\right)}-r \lambda_{X} e^{r t}\left(t-t_{y}\right)-\lambda_{X} e^{r t} \\
& =e^{r\left(t-t_{y}\right)}\left[\left(r \lambda_{K}^{0}+c_{K}\right) e^{r\left(t_{y}-t_{K}\right)}+c_{y}-c_{x}-\lambda_{X} e^{r t_{y}}\right]-r \lambda_{X} e^{r t}\left(t-t_{y}\right) .
\end{aligned}
$$


Next remark that $\left(r \lambda_{K}^{0}+c_{K}\right) e^{r\left(t_{y}-t_{K}\right)}+c_{y}-c_{x}-\lambda_{X} e^{r t_{y}}=\pi_{0} e^{r\left(t_{y}-t_{K}\right)}$ since $c_{y}=c_{x}+\lambda_{X} e^{r t_{y}}$. Thus:

$$
\frac{\partial \lambda_{K}^{2}(t)}{\partial t}=\pi_{0} e^{r\left(t-t_{K}\right)}-r \lambda_{X} e^{r t}\left(t-t_{y}\right)
$$

Furthermore:

$$
\begin{aligned}
\frac{\partial \lambda_{K}^{2}}{\partial t_{y}} & =e^{r\left(t-t_{y}\right)}\left[\lambda_{X} e^{r t_{y}}+c_{x}-c_{y}\right]=0 \\
\frac{\partial \lambda_{K}^{2}}{\partial t_{K}} & =-\pi_{0} e^{r\left(t-t_{K}\right)}<0 \\
\frac{\partial \lambda_{K}^{2}}{\partial \lambda_{X}} & =-e^{r t}\left(t-t_{y}\right)<0 \\
\frac{\partial \lambda_{K}^{2}(t)}{\partial \lambda_{K}^{0}} & =e^{r\left(t-t_{K}\right)}
\end{aligned}
$$

\section{A.3.2 Proof that the system (5.1)-(5.3) has a unique solution}

We now check that for $t_{K}=0$ and $\lambda_{K}(0)=\lambda_{K}^{0}$, the system (5.1)-(5.3) determines in a unique way the vector $\left(T, \lambda_{X}^{0}, K_{X}\right)$. Making use of (A.3.3) evaluated at $t_{K}=0$ and at $t_{X}=T$ yields the following expression of (5.2):

$$
\begin{aligned}
\lambda_{K}^{X}\left(K_{X}\right)= & \lambda_{K}(T)=\lambda_{K}^{0} e^{r T}+\frac{c_{K}}{r}\left(e^{r T}-1\right) \\
& +\frac{c_{y}-c_{x}}{r}\left(e^{r\left(T-t_{y}\right)}-1\right)-\lambda_{X}^{0} e^{r T}\left(T-t_{y}\right) .
\end{aligned}
$$

Denote by:

$$
\begin{aligned}
\left|p^{d^{\prime}}\right| & \equiv\left|\frac{d p^{d}(q)}{d q}\right|_{q=q(T)} \text { and }\left|\lambda_{K}^{X^{\prime}}\right| \equiv\left|\frac{d \lambda_{X}^{K}(K)}{d K}\right|_{K=K_{X}} \\
k^{s^{\prime}} & \equiv \frac{d k^{s}\left(\lambda_{K}\right)}{d \lambda_{K}}>0 .
\end{aligned}
$$

Differentiating the system (5.1)-(5.3) yields:

$$
\begin{aligned}
r \lambda_{X}^{0} d T+d \lambda_{X}^{0}+\left|p^{d^{\prime}}\right| e^{-r T} d K_{X} & =0 \\
\pi d T-\left(T-t_{y}\right) d \lambda_{X}^{0}+\left|\lambda_{K}^{X^{\prime}}\right| e^{-r T} d K_{X} & =-d \lambda_{K}^{0} \\
k(T) d T-J_{K}^{\lambda} d \lambda_{X}^{0}-d K_{X} & =-A_{K} d \lambda_{K}^{0}
\end{aligned}
$$


where:

$$
\begin{aligned}
& \pi \equiv r \lambda_{K}^{0}+c_{K}-r \lambda_{X}^{0}\left(T-t_{y}\right)=\pi_{0}-r \lambda_{X}^{0}\left(T-t_{y}\right)=\dot{\lambda}_{K}^{2}(T)<0 \\
& J_{K}^{\lambda} \equiv \int_{t_{y}}^{T} k^{s^{\prime}} e^{r t}\left(t-t_{y}\right) d t>0 \quad ; \quad A_{K} \equiv \int_{0}^{T} k^{s^{\prime}} e^{r t} d t>0 .
\end{aligned}
$$

It is immediately checked that when $t_{y}>0$, the $d t_{y}$ terms in the differentiation of the system (5.1)-(5.3) vanish. The linearized system is thus independent from $d t_{y}$.

Let $\Delta$ be the determinant of the linearized system (A.3.9)-(A.3.11):

$$
\begin{aligned}
\Delta=[ & \left.k(T)\left(T-t_{y}\right)-\pi J_{K}^{\lambda}\right]\left|p^{d^{\prime}}\right| e^{-r T}+\left[k(T)+r \lambda_{X}^{0} J_{K}^{\lambda}\right]\left|\lambda_{K}^{X^{\prime}}\right| e^{-r T} \\
& +\left[\pi+r \lambda_{X}^{0}\left(T-t_{y}\right)\right] .
\end{aligned}
$$

Since $\pi=\pi_{0}-r \lambda_{X}^{0}\left(T-t_{y}\right)$ :

$$
\begin{aligned}
\Delta=[ & \left.k(T)\left(T-t_{y}\right)-\pi J_{K}^{\lambda}\right]\left|p^{d^{\prime}}\right| e^{-r T}+\left[k(T)+r \lambda_{X}^{0} J_{K}^{\lambda}\right]\left|\lambda_{K}^{X^{\prime}}\right| e^{-r T} \\
& +\pi_{0} .
\end{aligned}
$$

Since $\pi<0, \Delta\rangle 0$. Hence the system (5.1)-(5.3) evaluated at $t_{K}=0$ defines a unique vector $\left(T, \lambda_{X}^{0}, K_{X}\right)$.

\section{A.3.3 Sensitivity of $\left(T, \lambda_{X}^{0}, K_{X}\right)$ with respect to $\lambda_{K}^{0}$}

Applying Cramer rule:

$$
\begin{aligned}
\frac{d T}{d \lambda_{K}^{0}} & =\frac{1}{\Delta}\left|\begin{array}{ccc}
0 & 1 & \left|p^{d^{\prime}}\right| e^{-r T} \\
-1 & -\left(T-t_{y}\right) & \left|\lambda_{K}^{X^{\prime}}\right| e^{-r T} \\
-A_{K} & -J_{K}^{\lambda} & -1
\end{array}\right| \\
& =\frac{1}{\Delta}\left\{\left[J_{K}^{\lambda}-A_{K}\left(T-t_{y}\right)\right]\left|p^{d^{\prime}}\right| e^{-r T}-A_{K}\left|\lambda_{K}^{X^{\prime}}\right| e^{-r T}-1\right\} \\
& =-\frac{1}{\Delta}\left\{1+\left[\left(T-t_{y}\right) A_{K}-J_{K}^{\lambda}\right]\left|p^{d^{\prime}}\right| e^{-r T}+A_{K}\left|\lambda_{K}^{X^{\prime}}\right| e^{-r T}\right\} .
\end{aligned}
$$


Since $t \leq T$ and $t_{y} \geq 0$ imply together that:

$$
\begin{aligned}
J_{K}^{\lambda} & =\int_{t_{y}}^{T} k^{s^{\prime}} e^{r t}\left(t-t_{y}\right) d t<\left(T-t_{y}\right) \int_{t_{y}}^{T} k^{s^{\prime}} e^{r t} d t \\
& \leq\left(T-t_{y}\right) \int_{0}^{T} k^{s^{\prime}} e^{r t} d t=\left(T-t_{y}\right) A_{K}
\end{aligned}
$$

it results that $d T / d \lambda_{K}^{0}<0$.

Next:

$$
\begin{aligned}
\frac{d \lambda_{X}^{0}}{d \lambda_{K}^{0}} & =\frac{1}{\Delta}\left|\begin{array}{ccc}
r \lambda_{X}^{0} & 0 & \left|p^{d^{\prime}}\right| e^{-r T} \\
\pi & -1 & \left|\lambda_{K}^{X^{\prime}}\right| e^{-r T} \\
k(T) & -A_{K} & -1
\end{array}\right| \\
& =\frac{1}{\Delta}\left\{\left[k(T)-\pi A_{K}\right]\left|p^{d^{\prime}}\right| e^{-r T}+r \lambda_{X}^{0} A_{K}\left|\lambda_{K}^{X^{\prime}}\right| e^{-r T}+r \lambda_{X}^{0}\right\}>0 .
\end{aligned}
$$

Making use of (A.3.9) together with the above expressions of $d T / d \lambda_{K}^{0}$ and $d \lambda_{X}^{0} / d \lambda_{K}^{0}$, the following expression of $d K_{X} / d \lambda_{K}^{0}$ results:

$$
\begin{aligned}
\frac{d K_{X}}{d \lambda_{K}^{0}}\left|p^{d^{\prime}}\right| e^{-r T}= & r \lambda_{X}^{0} \frac{d T}{d \lambda_{K}^{0}}+\frac{d \lambda_{X}^{0}}{d \lambda_{K}^{0}} \\
= & \frac{1}{\Delta}\left\{-r \lambda_{X}^{0}\left[1+\left(\left(T-t_{y}\right) A_{K}-J_{K}^{\lambda}\right)\left|p^{d^{\prime}}\right| e^{-r T}+A_{K}\left|\lambda_{K}^{X^{\prime}}\right| e^{-r T}\right]\right. \\
& \left.+\left[\left(k(T)-\pi A_{K}\right)\left|p^{d^{\prime}}\right| e^{-r T}+r \lambda_{X}^{0} A_{K}\left|\lambda_{K}^{X^{\prime}}\right| e^{-r T}+r \lambda_{X}^{0}\right]\right\} \\
= & \frac{1}{\Delta}\left\{k(T)-\pi A_{K}-r \lambda_{X}^{0}\left(T-t_{y}\right) A_{K}+r \lambda_{X}^{0} J_{K}^{\lambda}\right\}\left|p^{d^{\prime}}\right| e^{-r T} .
\end{aligned}
$$

The HJB equation implies that $d K_{X} / d \lambda_{K}^{0}=0$. Since $\pi=\pi_{0}-r \lambda_{X}^{0}\left(T-t_{y}\right)$, it thus results that:

$$
k(T)-\pi_{0} A_{K}+r \lambda_{X}^{0} J_{K}^{\lambda}=0 .
$$

Next, making use of $\pi=\pi_{0}-r \lambda_{X}^{0}\left(T-t_{y}\right)$ yields the following equivalent expression of $\Delta$ :

$$
\begin{gathered}
\Delta=[( \\
\left.\left.\Delta(T)+r \lambda_{X}^{0} J_{K}^{\lambda}\right)\left(T-t_{y}\right)-\pi_{0} J_{K}^{\lambda}\right]\left|p^{d^{\prime}}\right| e^{-r T} \\
+\left[k(T)+r \lambda_{X}^{0} J_{K}^{\lambda}\right]\left|\lambda_{K}^{X^{\prime}}\right| e^{-r T}+\pi_{0} .
\end{gathered}
$$


Taking (A.3.12) into account:

$$
\begin{aligned}
\Delta & =\left[\pi_{0} A_{K}\left(T-t_{y}\right)-\pi_{0} J_{K}^{\lambda}\right]\left|p^{d^{\prime}}\right| e^{-r T}+\pi_{0} A_{K}\left|\lambda_{K}^{X^{\prime}}\right| e^{-r T}+\pi_{0} \\
& =\pi_{0}\left\{1+\left[A_{K}\left(T-t_{y}\right)-J_{K}^{\lambda}\right]\left|p^{d^{\prime}}\right| e^{-r T}+A_{K}\left|\lambda_{K}^{X^{\prime}}\right| e^{-r T}\right\} .
\end{aligned}
$$

It results from (A.3.13) that:

$$
\frac{d T}{d \lambda_{K}^{0}}=-\frac{1}{\Delta} \frac{\Delta}{\pi_{0}}=-\frac{1}{\pi_{0}} .
$$

Since $d K_{X} / d \lambda_{K}^{0}=0$, the price condition (A.3.9) at $T$ implies together with (A.3.14) that:

$$
\frac{d \lambda_{X}^{0}}{d \lambda_{K}^{0}}=-r \lambda_{X}^{0} \frac{d T}{d \lambda_{K}^{0}}=\frac{r \lambda_{X}^{0}}{\pi_{0}} .
$$

Last, if $t_{y}>0$, the condition: $c_{y}=c_{x}+\lambda_{X}^{0} e^{r t_{y}}$ shows that $r \lambda_{X}^{0} d t_{y}=-d \lambda_{X}^{0}$. Thus:

$$
\frac{d t_{y}}{d \lambda_{K}^{0}}=-\frac{1}{r \lambda_{X}^{0}} \frac{d \lambda_{X}^{0}}{d \lambda_{K}^{0}}=-\frac{1}{\pi_{0}}=\frac{d T}{d \lambda_{K}^{0}}<0 .
$$

The active solar production phase length, $T-t_{y}$, is independent from $\lambda_{K}^{0}$ in scenarios of type 1 or 3 .

\section{A.3.4 Proof that $d X^{0}\left(\lambda_{K}^{0}\right) / d \lambda_{K}^{0}<0$}

An equivalent expression of (5.4) is:

$$
X^{0}\left(\lambda_{K}^{0}\right)=\int_{0}^{T} q^{d}\left(c_{x}+\lambda_{X}^{0} e^{r t}\right) d t-\int_{t_{y}}^{T} K(t) d t
$$

Since $\dot{K}(t)=k(t)$ and letting $K\left(t_{y}\right) \equiv K_{y}, K(t), t \in\left(t_{y}, T\right)$, is given by:

$$
K(t)=K_{y}+\int_{t_{y}}^{t} k(\tau) d \tau .
$$

Hence:

$$
\int_{t_{y}}^{T} K(t) d t=K_{y}\left(T-t_{y}\right)+\int_{t_{y}}^{T} \int_{t_{y}}^{t} k(\tau) d \tau d t
$$


Inverting the integration order:

$$
\int_{t_{y}}^{T} K(t) d t=\left(T-t_{y}\right) \int_{0}^{t_{y}} k(t) d t+\int_{t_{y}}^{T} k(t)(T-t) d t .
$$

The following expression of $X^{0}\left(\lambda_{K}^{0}\right)$ results:

$$
\begin{aligned}
X^{0}\left(\lambda_{K}^{0}\right)= & \int_{0}^{T} q^{d}\left(c_{x}+\lambda_{X}^{0} e^{r t}\right) d t-\left(T-t_{y}\right) \int_{0}^{t_{y}} k^{s}\left(\lambda_{K}^{1}(t)\right) d t \\
& -\int_{t_{y}}^{T} k^{s}\left(\lambda_{K}^{2}(t)\right)(T-t) d t
\end{aligned}
$$

Next differentiating the cumulated oil consumption constraint (A.3.17) yields:

$$
\begin{aligned}
d X^{0}= & q(T) d T+\int_{0}^{T} \frac{d q^{d}(p(t))}{d p(t)} e^{r t} d t d \lambda_{X}^{0} \\
& -\left(d T-d t_{y}\right) \int_{0}^{t_{y}} k^{s}(t) d t-\left(T-t_{y}\right) k^{s}\left(t_{y}\right) d t_{y}-\left(T-t_{y}\right) \int_{0}^{t_{y}} k^{s^{\prime}} \frac{\partial \lambda_{K}^{1}}{\partial \lambda_{K}^{0}} d t d \lambda_{K}^{0} \\
& +\left(T-t_{y}\right) k^{s}\left(t_{y}\right) d t_{y}-\int_{t_{y}}^{T} k^{s^{\prime}}\left[\frac{\partial \lambda_{K}^{2}(t)}{\partial \lambda_{K}^{0}} d \lambda_{K}^{0}+\frac{\partial \lambda_{K}^{2}(t)}{\partial \lambda_{X}^{0}} d \lambda_{X}^{0}\right](T-t) d t \\
& -\int_{t_{y}}^{T} k^{s}(t) d t d T .
\end{aligned}
$$

Denote by $q^{d^{\prime}}(t) \equiv d q^{d}(p(t)) / d p(t)$. Simplifying and rearranging yields:

$$
\begin{aligned}
d X^{0}= & {\left[q(T)-\int_{0}^{T} k^{s}(t) d t\right] d T+K_{y} d t_{y} } \\
& +\left[\int_{0}^{T} q^{d^{\prime}} e^{r t} d t-\int_{t_{y}}^{T} k^{s^{\prime}} \frac{\partial \lambda_{K}^{2}(t)}{\partial \lambda_{X}^{0}}(T-t) d t\right] d \lambda_{X}^{0} \\
& -\left[\left(T-t_{y}\right) \int_{0}^{t_{y}} k^{s^{\prime}} \frac{\partial \lambda_{K}^{1}(t)}{\partial \lambda_{K}^{0}} d t+\int_{t_{y}}^{T} k^{s^{\prime}} \frac{\partial \lambda_{K}^{2}(t)}{\partial \lambda_{K}^{0}}(T-t) d t\right] d \lambda_{K}^{0} .
\end{aligned}
$$

Since $q(T)=K_{X}=\int_{0}^{T} k^{s}(t) d t$ and taking (A.3.7) into account:

$$
\begin{aligned}
d X^{0}= & K_{y} d t_{y}+\left[\int_{0}^{T} q^{d^{\prime}} e^{r t} d t+\int_{t_{y}}^{T} k^{s^{\prime}} e^{r t}\left(t-t_{y}\right)(T-t) d t\right] d \lambda_{X}^{0} \\
& -\left[\left(T-t_{y}\right) \int_{0}^{t_{y}} k^{s^{\prime}} e^{r t} d t+\int_{t_{y}}^{T} k^{s^{\prime}} e^{r t}(T-t) d t\right] d \lambda_{K}^{0} .
\end{aligned}
$$


Remark that $c_{y}=c_{x}+\lambda_{X}^{0} e^{r t_{y}}$ implies that $d t_{y}=-d \lambda_{X}^{0} / r \lambda_{X}^{0}$. Taking the expression (A.3.15) of $d \lambda_{X}^{0} / d \lambda_{K}^{0}$ into account yields:

$$
\begin{aligned}
\frac{d X^{0}}{d \lambda_{K}^{0}}= & \frac{r \lambda_{X}^{0}}{\pi_{0}} \int_{0}^{T} q^{d^{\prime}} e^{r t} d t-\frac{K_{y}}{\pi_{0}}+\int_{t_{y}}^{T} k^{s^{\prime}} \frac{r \lambda_{X}^{0}}{\pi_{0}} e^{r t}\left(t-t_{y}\right)(T-t) d t \\
& -\left(T-t_{y}\right) \int_{0}^{t_{y}} k^{s^{\prime}} e^{r t} d t-\int_{t_{y}}^{T} k^{s^{\prime}} e^{r t}(T-t) d t
\end{aligned}
$$

Since $\dot{\lambda}_{K}^{2}(t)=\pi_{0} e^{r t}-r \lambda_{X}^{0} e^{r t}\left(t-t_{y}\right), t \in\left[t_{y},\right]$, the above is equivalent to:

$$
\begin{aligned}
\frac{d X^{0}}{d \lambda_{K}^{0}}= & \frac{r \lambda_{X}^{0}}{\pi_{0}} \int_{0}^{T} q^{d^{\prime}} e^{r t} d t-\frac{K_{y}}{\pi_{0}}-\frac{1}{\pi_{0}} \int_{t_{y}}^{T} k^{s^{\prime}} \dot{\lambda}_{K}^{2}(t)(T-t) d t \\
& -\left(T-t_{y}\right) \int_{0}^{t_{y}} k^{s^{\prime}} e^{r t} d t \\
= & \frac{r \lambda_{X}^{0}}{\pi_{0}} \int_{0}^{T} q^{d^{\prime}} e^{r t} d t-\frac{K_{y}}{\pi_{0}}-\frac{1}{\pi_{0}} \int_{t_{y}}^{T} \dot{k}^{s}(t)(T-t) d t \\
& -\left(T-t_{y}\right) \int_{0}^{t_{y}} k^{s^{\prime}} e^{r t} d t
\end{aligned}
$$

Integrating by parts the second integral yields:

$$
\begin{aligned}
\int_{t_{y}}^{T} \dot{k}^{s}(t)(T-t) d t & =\left.k^{s}(t)(T-t)\right|_{t_{y}} ^{T}+\int_{t_{y}}^{T} k^{s}(t) d t \\
& =-k^{s}\left(t_{y}\right)\left(T-t_{y}\right)+\int_{t_{y}}^{T} k^{s}(t) d t .
\end{aligned}
$$

Thus:

$$
\begin{aligned}
\frac{d X^{0}}{d \lambda_{K}^{0}}= & \frac{r \lambda_{X}^{0}}{\pi_{0}} \int_{0}^{T} q^{d^{\prime}} e^{r t} d t-\frac{K_{y}}{\pi_{0}}+\frac{k^{s}\left(t_{y}\right)\left(T-t_{y}\right)}{\pi_{0}}-\frac{1}{\pi_{0}} \int_{t_{y}}^{T} k(t) d t \\
& -\left(T-t_{y}\right) \int_{0}^{t_{y}} k^{s^{\prime}} e^{r t} d t \\
= & \frac{1}{\pi_{0}}\left\{r \lambda_{X}^{0} \int_{0}^{T} q^{d^{\prime}} e^{r t} d t-\left[K_{y}+\int_{t_{y}}^{T} k(t) d t\right]\right\} \\
& +\frac{\left(T-t_{y}\right)}{\pi_{0}}\left[k^{s}\left(t_{y}\right)-\pi_{0} \int_{0}^{t_{y}} k^{s^{\prime}} e^{r t} d t\right] \\
= & \frac{1}{\pi_{0}}\left\{r \lambda_{X}^{0} \int_{0}^{T} q^{d^{\prime}} e^{r t} d t-K_{X}\right\}+\frac{\left(T-t_{y}\right)}{\pi_{0}}\left[k^{s}\left(t_{y}\right)-\pi_{0} \int_{0}^{t_{y}} k^{s^{\prime}} e^{r t} d t\right] .
\end{aligned}
$$


Since $\dot{p}(t)=r \lambda_{X}^{0} e^{r t}$ over the time interval $[0, T)$ :

$$
\begin{aligned}
\int_{0}^{T} q^{d^{\prime}} e^{r t} d t & =\frac{1}{r \lambda_{X}^{0}} \int_{0}^{T} q^{d^{\prime}}(p(t)) \dot{p}(t) d t=\frac{1}{r \lambda_{X}^{0}} \int_{0}^{T} \dot{q}^{d}(t) d t \\
& =\frac{1}{r \lambda_{X}^{0}}(q(T)-q(0)) .
\end{aligned}
$$

Thus $q(T)=K_{X}$ implies that:

$$
\frac{d X^{0}}{d \lambda_{K}^{0}}=-\frac{q(0)}{\pi_{0}}+\frac{\left(T-t_{y}\right)}{\pi_{0}}\left[k^{s}\left(t_{y}\right)-\pi_{0} \int_{0}^{t_{y}} k^{s^{\prime}} e^{r t} d t\right] .
$$

The relation (A.3.12) resulting from the HJB equation yields:

$$
\begin{aligned}
k(T) & =\pi_{0} A_{K}-r \lambda_{X}^{0} J_{K}^{\lambda} \\
& =\pi_{0} \int_{0}^{t_{y}} k^{s^{\prime}} e^{r t} d t+\pi_{0} \int_{t_{y}}^{T} k^{s^{\prime}} e^{r t} d t-r \lambda_{X}^{0} \int_{t_{y}}^{T} k^{s^{\prime}} e^{r t}\left(t-t_{y}\right) d t \\
& =\pi_{0} \int_{0}^{t_{y}} k^{s^{\prime}} e^{r t} d t+\int_{t_{y}}^{T}\left[\pi_{0}-r \lambda_{X}^{0}\left(t-t_{y}\right)\right] e^{r t} d t .
\end{aligned}
$$

Making use once again of the expression (A.3.4) of $\dot{\lambda}_{K}^{2}(t)$ :

$$
\begin{aligned}
k(T) & =\pi_{0} \int_{0}^{t_{y}} k^{s^{\prime}} e^{r t} d t+\int_{t_{y}}^{T} k^{s^{\prime}} \dot{\lambda}_{K}^{2}(t) d t \\
& =\pi_{0} \int_{0}^{t_{y}} k^{s^{\prime}} e^{r t} d t+\int_{t_{y}}^{T} \dot{k}(t) d t \\
& =\pi_{0} \int_{0}^{t_{y}} k^{s^{\prime}} e^{r t} d t+k(T)-k^{s}\left(t_{y}\right) .
\end{aligned}
$$

Hence:

$$
k^{s}\left(t_{y}\right)=\pi_{0} \int_{0}^{t_{y}} k^{s^{\prime}} e^{r t} d t
$$

implying that:

$$
\frac{d X^{0}}{d \lambda_{K}^{0}}=-\frac{q(0)}{\pi_{0}}<0
$$




\section{A.4 Appendix A.4}

The argument is the following. In order that the purchase price $p_{K}(t)$ be constant at $p_{K}(t)=c_{s}$ and simultaneously that the investment rate $k(t)$ be positive over a time interval $\left(t_{1}, t_{2}\right), t_{1}<t_{2}$, we should have through $(2.3)$ and (2.6) both holding:

$$
p_{K}(t)=\lambda_{K}(t)=c_{s} \Longrightarrow \dot{\lambda}_{K}(t)=0=r c_{s}+c_{K}-\gamma_{K}(t), t \in\left(t_{1}, t_{2}\right) .
$$

The last equality comes from $K(t)>0$, hence $\nu_{K}=0: k(t)>0$ implies that $K(t)$ increases within the interval, hence is positive.

From (2.2) which also holds, and (A.4.1), we get:

$$
\gamma_{K}(t)=p(t)-c_{y}-\gamma_{y}(t)=r c_{s}+c_{K}>0 .
$$

Since $\gamma_{y}(t) \geq 0$, (A.4.2) cannot hold during any investment phase within which the solar energy production is nil and $K(t)>0$. A necessary condition for $y(t)=0$ when $K(t)>0$ is that $p(t) \leq c_{y}$, hence $p(t)-c_{y}-\gamma_{y}(t) \leq 0$.

For an investment phase during which both fossil and solar energies are simultaneously exploited, $p(t)=c_{x}+\lambda_{X} e^{r t}$ and $\gamma_{y}(t)=0$, thus $\gamma_{K}(t)$ must increase and again (A.4.2) cannot hold. Last assume that the solar production is positive, $\gamma_{y}(t)=0$, that oil is exhausted and that (A.4.2) holds, that is: $p(t)-c_{y}=r c_{s}+c_{K}>0$. Since $p(t)>c_{y}$ then the available solar equipment $K(t)$ is wholly exploited: $p(t)=p^{d}(K(t))$. Since $k(t)>0$, then $\dot{p}(t)=p^{d^{\prime}}(K(t)) k(t)<0$, hence $\dot{\gamma}_{K}(t)<0$, a contradiction. 


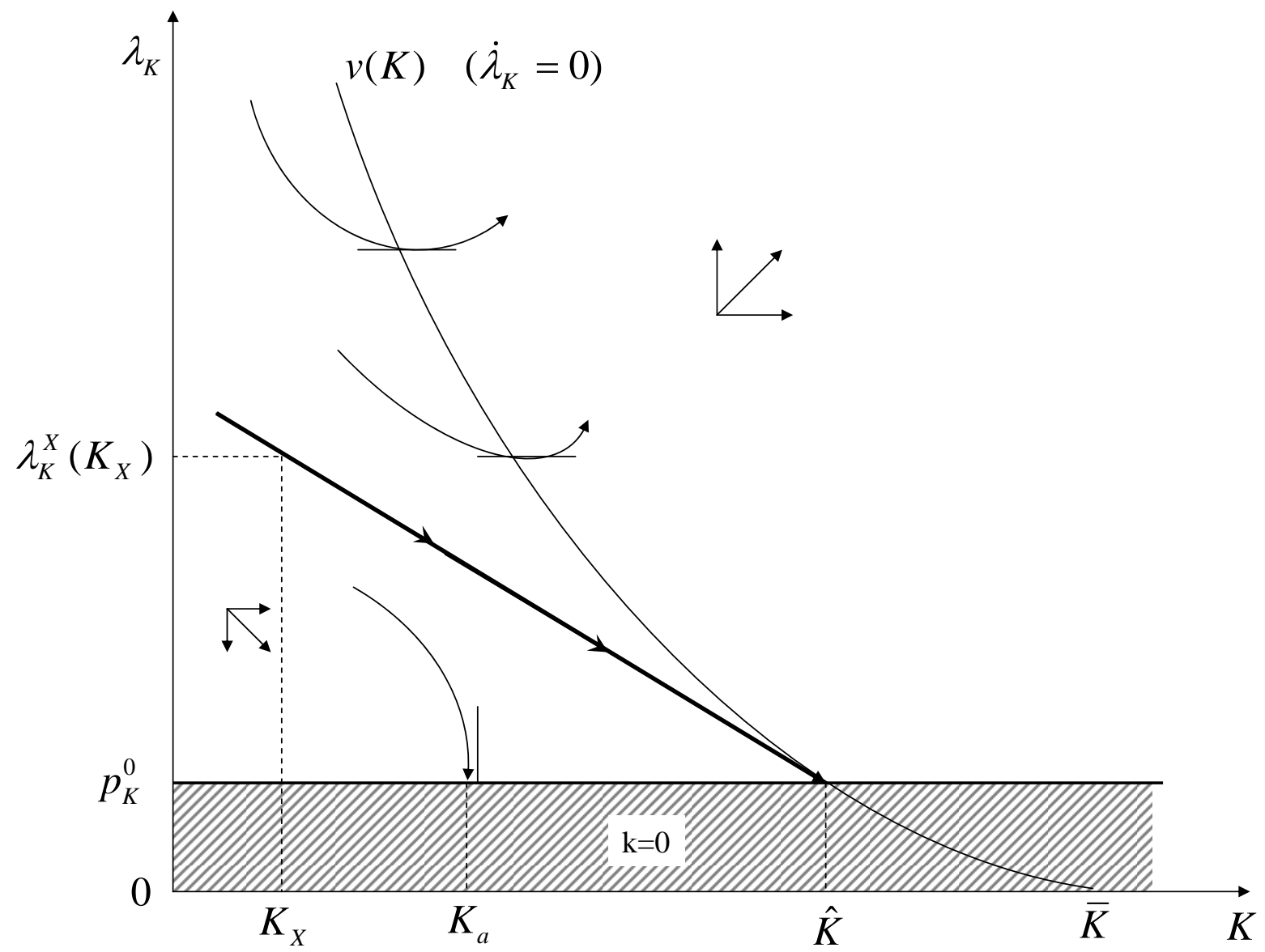

Figure 1: Equilibrium Solar Capital Expansion Path after Oil Depletion. 


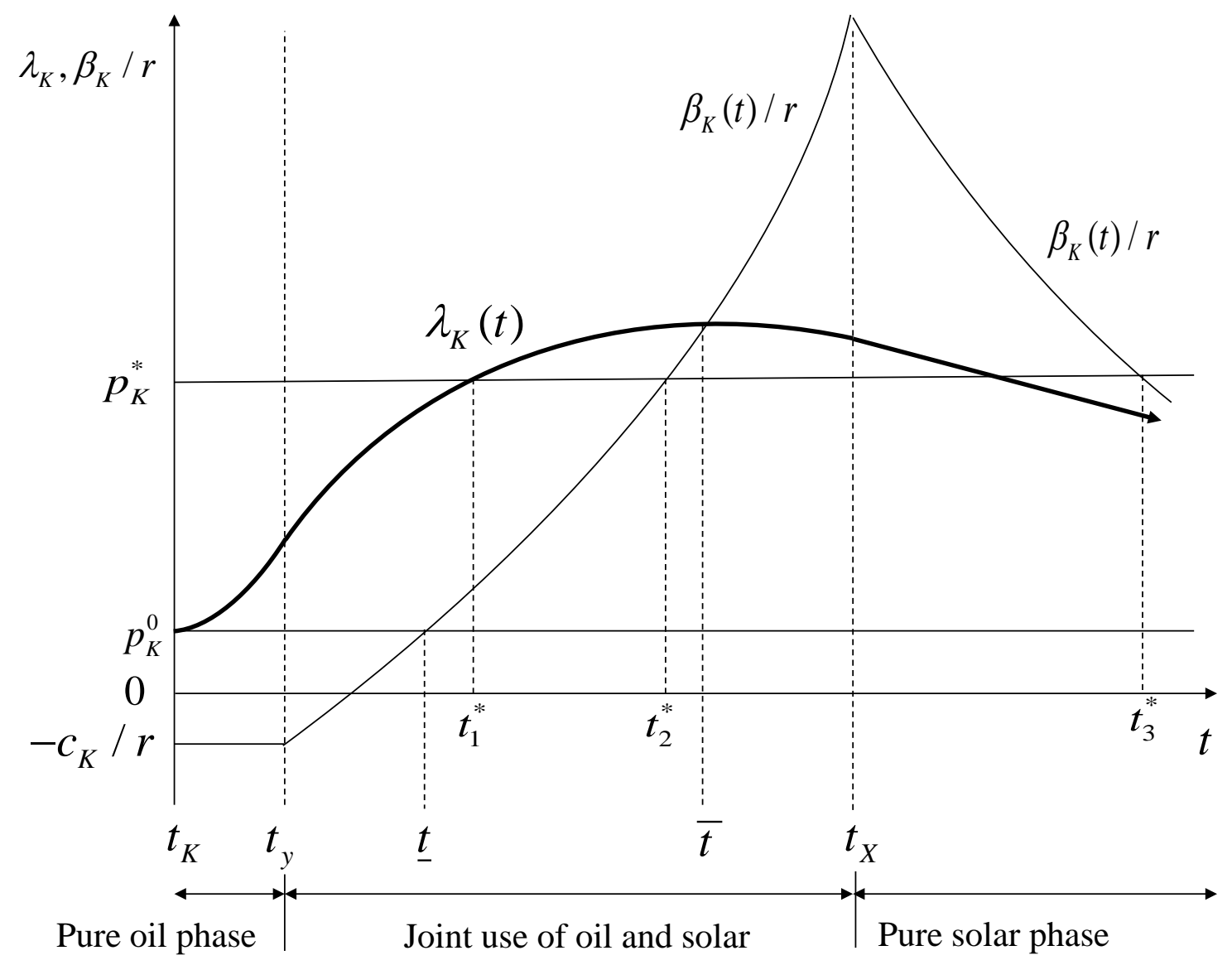

Figure 2: Dynamics of $\lambda_{K}$ and $\beta_{K}$. Scenario 1: $0<t_{K}<t_{y}$. 

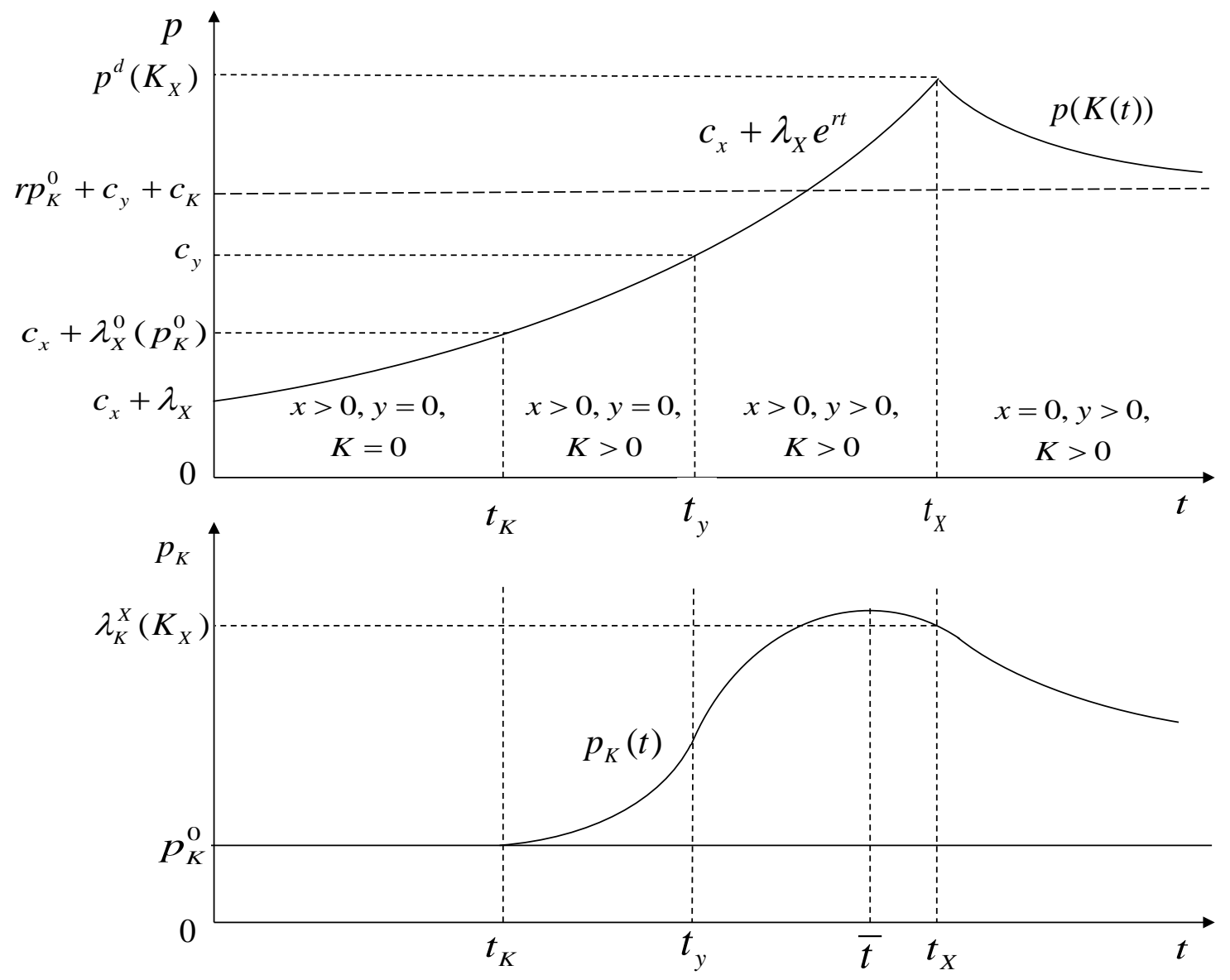

Figure 3: Energy Price and Solar Equipment Price Dynamics. Scenario 1 with $0<t_{K}<t_{y}$. 